\title{
LQR CONTROL OF THIN SHELL DYNAMICS: FORMULATION AND NUMERICAL IMPLEMENTATION ${ }^{1}$
}

\author{
R.C.H. del Rosario \\ Center for Research in Scientific Computation \\ North Carolina State University \\ Raleigh, NC 27695 \\ rcdelros@eos.ncsu.edu \\ R.C. Smith \\ Department of Mathematics \\ Iowa State University \\ Ames, IA 50011 \\ rsmith@iastate.edu
}

\begin{abstract}
A PDE-based feedback control method for thin cylindrical shells with surface-mounted piezoceramic actuators is presented. Donnell-Mushtari equations modified to incorporate both passive and active piezoceramic patch contributions are used to model the system dynamics. The well-posedness of this model and the associated LQR problem with an unbounded input operator are established through analytic semigroup theory. The model is discretized using a Galerkin expansion with basis functions constructed from Fourier polynomials tensored with cubic splines, and convergence criteria for the associated approximate LQR problem are established. The effectiveness of the method for attenuating the coupled longitudinal, circumferential and transverse shell displacements is illustrated through a set of numerical examples.
\end{abstract}

\footnotetext{
${ }^{1}$ This research was supported in part by the National Aeronautics and Space Administration under NASA Contract Number NAS1-19480 while RCS was a visiting scientist at the Institute for Computer Applications in Science and Engineering (ICASE), NASA Langley Research Center, Hampton, VA 23681. Additional support was also provided in part under NASA grant NAG-1-1600.
} 


\section{Introduction}

Thin shell models are used to characterize structural phenomena ranging from wing vibrations to deformations in a duct due to an adjacent flow field. In full generality, shell equations can be used to model structures with variable curvature and irregular geometries. Furthermore, the models can be modified to incorporate a large variety of actuators and sensors and can be coupled with adjacent acoustic or fluid fields to model coupled systems. In all cases, the displacements of various shell components are coupled due to the curvature and geometry.

We focus here on cylindrical shell models due to their prevalence in applications (e.g., noise control in a fuselage or flow control in a flexible pipe). For specificity, we consider surfacemounted piezoceramic actuators due to their capability for both sensing and actuating. Their applicability is enhanced by the fact that their response is relatively linear at low to moderate drive levels. Furthermore, they are lightweight, space efficient, and can be manufactured for various geometries at reasonable cost. We note that while the control methods discussed here are specific to surface-mounted piezoceramic actuators, the techniques can be directly extended to embedded piezoceramic actuators as well as electrostrictive or magnetostrictive actuators which have been restricted to output levels which are approximately linear.

While experimental work has demonstrated the potential of piezoceramic actuators in cylindrical shell applications $[13,25]$, their full potential is typically not realized due to limitations in hardware, models, approximation methods and control laws. One source of difficulty when approximating shell dynamics and designing model-based controllers is due to the mechanisms which provide the models with much of their flexibility, namely the coupling between shell components. The physical coupling due to geometry and curvature leads to a significant interlacing of frequencies (e.g., as illustrated in [12], it is common in certain regimes to have ten or more linearly independent modes within a $10 \mathrm{~Hz}$ range). This places stringent requirements on the accuracy of approximation methods and the ability of the controller to avoid spillover of energy into unmodeled mechanisms.

One means of approximation in structural applications is through modal or eigenfunction expansions. Because analytic expressions for eigenfunctions can be determined only for very restrictive boundary conditions (e.g., simply supported) for shells with constant material parameters and hence no actuators, sensors or coupling components (normal modes do not exist for shells with piecewise constant parameters [6]), approximation techniques or assumed modal expansions must be employed. The coupling and consequent interlacing of frequencies places stringent requirements on the accuracy of approximation methods or choice of assumed modal basis. The effects of coupling must also be considered when designing a feedback control method which employs piezoceramic patches. For example, the tight coupling and subsequent frequency interlacing can cause a modal controller constructed solely for resolution of uncontrolled dynamics to experience severe spillover into uncontrolled modes upon application of the control input. This motivates the development of a more general model-based control method for shell applications.

In this paper, we consider an LQR full state feedback law for cylindrical shells modeled by Donnell-Mushtari equations modified to account for actuator dynamics. An approximate feedback law appropriate for numerical implementation is developed in the context of a Fourier/spline-based Galerkin method. The Donnell-Mushtari equations characterize the coupling and physics in a large number of thin shell applications. Furthermore, the analysis 
and approximation methods developed in this context can be directly extended to more accurate models (e.g., Byrne-Flügge-Lur'ye model) if the application warrants. The consideration of LQR performance illustrates properties of the system and model-based control techniques and facilitates investigations regarding issues such as patch number and configuration. While full state measurements are not available using current instrumentation, and hence the techniques cannot directly be implemented in experiments, they provide an important first step in the design of effective compensators based on state estimates calculated using a limited number of observations (see [10]). The consideration of the LQR problem also provides a step toward the development of model-based controllers for fully coupled structural acoustic and fluid/structure systems involving cylindrical shells. As detailed in [12], the Galerkin approximation method employed here is flexible with respect to boundary conditions and facilitates consideration of the distributional derivatives which arise through the inclusion of patch contributions in the model. It also provides the accuracy required to resolve dynamics in the fully coupled model. From a control perspective, the use of the general spline basis eliminates the tendency toward spillover into unmodeled components of the system. Furthermore, the approximation method satisfies the adjoint convergence and maintenance of uniform decay bounds required for convergence of control gains. This enhances the robustness of the method in a variety of applications.

We note that additional theory concerning stabilization and feedback control of thin shells from a PDE perspective can be found in [17, 18, 22]. Furthermore, an alternative framework for establishing the convergence of control gains for the abstract operator formulation is given in $[16,19]$. Both sets of references are theoretical in nature, however, and do not provide numerical methods or simulation results. The difference and primary contribution of this paper lies in the comprehensive treatment of the problem in a framework which includes model development and well-posedness analysis, a rigorous LQR control formulation, incorporation of an efficient numerical method, and numerical examples demonstrating the scope of the method.

Strong and weak forms of the the thin shell model are summarized in Section 2. These models are obtained through modification of the classical Donnell-Mushtari equations to incorporate passive (material) and active (actuator) contributions due to the patches. The model is also posed in an abstract form based upon sesquilinear forms to provide a framework amenable to establishment of model well-posedness and development of an LQR method. Infinite dimensional LQR methods for systems with no exogenous disturbance or systems with a periodic disturbance are discussed in Section 3. In both cases, unbounded control inputs result due to the piecewise support of the patches. For the case with no exogenous disturbance, criteria guaranteeing the convergence of approximate suboptimal gains to optimal gains for the infinite dimensional system are established through application of analytic semigroup theory. The Fourier/spline-based Galerkin method of [12] is outlined in Section 4, and numerical examples demonstrating the control method are presented in Section 5. These example demonstrates that through the use of the model-based methodology with general Galerkin approximations, significant attenuation in shell vibrations can be obtained using piezoceramic actuators. 


\section{PDE Model}

Throughout this discussion, we consider a cylindrical shell whose radius is large in comparison with the thickness (see [15, 21] for details regarding limits on ratios). In accordance with common experimental clamping techniques, the edges are taken to be fixed in the sense that longitudinal, circumferential and transverse displacements along with transverse slopes are held fixed. Control is provided by surface-mounted piezoceramic patches which are configured in pairs with edges aligned with the circumferential and longitudinal axes of the shell.

To specify the geometry for the model, the longitudinal axis of the shell is taken to be aligned with the $x$-axis as depicted in Figure 1 . The length, thickness and radius of the shell are respectively denoted by $\ell, h$ and $R$ while the region occupied by the middle surface is denoted by $\Gamma_{0}$. The shell is assumed to have mass density $\rho_{s}$, Young's modulus E. Poisson ratio $\nu$, Kelvin-Voigt damping coefficient $c_{D}$ and air damping coefficient $\mu$. Note that the assumption of Kelvin-Voigt damping is reasonable and typical for many shell materials such as aluminum. Finally, the external surface forces are denoted by $\hat{q}_{x}, \hat{q}_{\theta}, \hat{q}_{n}$ while the displacements of the middle surface in the longitudinal, circumferential and transverse directions are denoted by $u, v$ and $w$, respectively.

It is assumed that $s$ pairs of piezoceramic patches having thickness $h_{p e}$, density $\rho_{p e}$, Young's modulus $E_{p e}$. Poisson ratio $\nu_{p e}$ and Kelvin-Voigt damping coefficient $c_{D_{p e}}$ are mounted to the inner and outer surfaces of the shell. Furthermore, it is assumed that the glue bonding layer provides negligible contribution to the structural dynamics (the reader is referred to $[8,10]$ for details concerning the incorporation of differing patch characteristics and bonding layers in the ensuing models).

\subsection{Strong Form of the Modeling Equation}

The dynamics of the previously described thin cylindrical shell with surface-mounted piezoceramic actuators are modeled by the modified Donnell-Mushtari equations

$$
\begin{array}{r}
R \rho h \frac{\partial^{2} u}{\partial t^{2}}-R \frac{\partial N_{x}}{\partial x}-\frac{\partial N_{\theta x}}{\partial \theta}=R \hat{q}_{x}-R \sum_{i=1}^{s} \frac{\partial\left(N_{x}\right)_{p e_{i}}}{\partial x} \mathcal{S}_{p e_{i}}(x, \theta) \\
R \rho h \frac{\partial^{2} v}{\partial t^{2}}-\frac{\partial N_{\theta}}{\partial \theta}-R \frac{N_{x \theta}}{\partial x}=R \hat{q}_{\theta}-\sum_{i=1}^{s} \frac{\partial\left(N_{\theta}\right)_{p e_{i}}}{\partial \theta} \mathcal{S}_{p e_{i}}(x, \theta) \\
R \rho h \frac{\partial^{2} w}{\partial t^{2}}+R \mu \frac{\partial w}{\partial t}-R \frac{\partial^{2} M_{x}}{\partial x^{2}}-\frac{1}{R} \frac{\partial^{2} M_{\theta}}{\partial \theta^{2}}-2 \frac{\partial^{2} M_{x \theta}}{\partial x \partial \theta}+N_{\theta} \\
=R \hat{q}_{n}-\sum_{i=1}^{s}\left[R \frac{\partial^{2}\left(M_{x}\right)_{p e_{i}}}{\partial x^{2}}+\frac{1}{R} \frac{\partial^{2}\left(M_{\theta}\right)_{p e_{i}}}{\partial \theta^{2}}\right]
\end{array}
$$

As detailed in $[8,10,21]$, equations of this type are obtained through force and moment balancing with only low order terms retained. Here $M_{x}, M_{\theta}, M_{\theta x}$ and $M_{x \theta}$ are internal moments, $N_{x}, N_{\theta}, N_{\theta x}$ and $N_{x \theta}$ denote internal force resultants, and $\left(M_{x}\right)_{p e_{i}},\left(M_{\theta}\right)_{p \epsilon_{i}},\left(N_{x}\right)_{p e_{i}},\left(N_{\theta}\right)_{p e_{i}}$ designate the external resultants (line moments and forces) generated by the $i^{t h}$ patch pair. The 


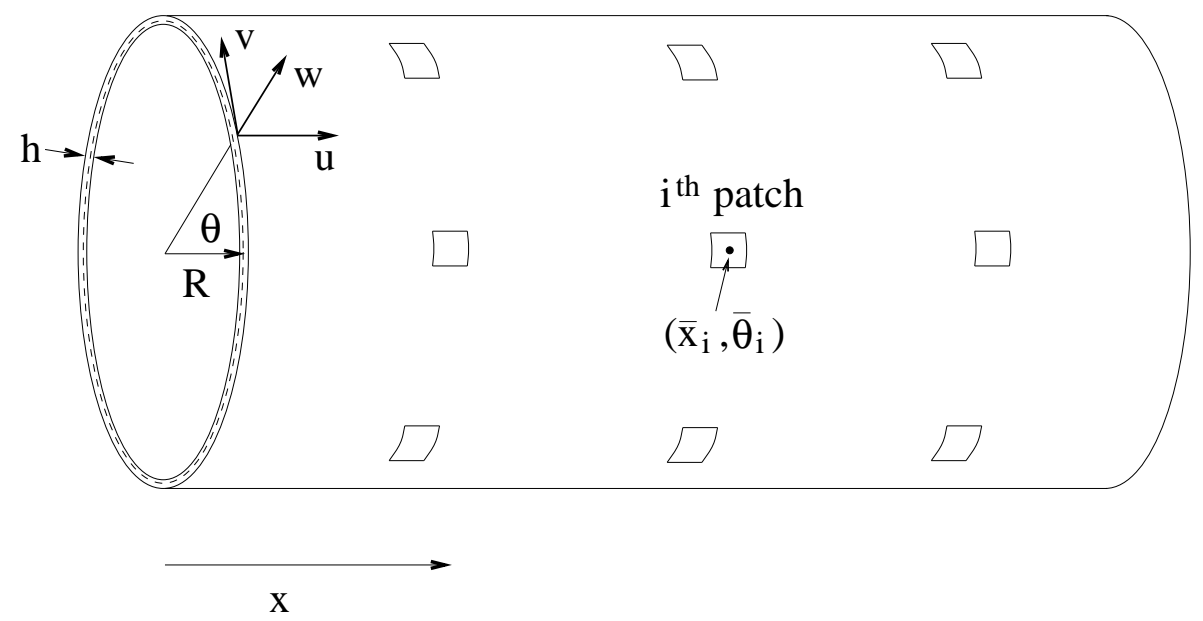

Figure 1. Thin cylindrical shell with surface mounted piezoceramic patches.

indicator function $\mathcal{S}_{p \epsilon_{i}}(x, \theta) \equiv S_{1,2}(x) \hat{S}_{1,2}(\theta)$, where

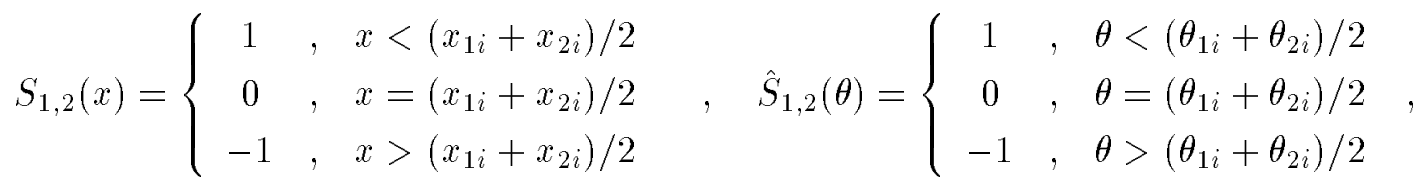

delineates the sense of the forces generated by the $i^{t h}$ pair. The symmetry of the function arises from the property that for homogeneous patches having uniform thickness, equal but opposite strains are generated about the point $\left(\bar{x}_{i}, \bar{\theta}_{i}\right)=\left(\left(x_{1 i}+x_{2 i}\right) / 2,\left(\theta_{1 i}+\theta_{2 i}\right) / 2\right)$.

The composite density $\rho$ consists of the shell density $\rho_{s}$ in regions devoid of patches and a linear combination of $\rho_{s}$ and the patch density $\rho_{p e}$ in regions covered by patchs (see [10] for details). Hence $\rho$ is piecewise constant with discontinuities at the patch edges.

Expressions for the internal force and moment resultants are derived under the assumption that stress is proportional to a linear combination of strain and strain rate. This yields a model which incorporates Kelvin-Voigt or strong internal damping. As detailed in [8,10], the resultants $N_{x}, N_{x \theta}, N_{\theta x}, M_{x}, M_{x \theta}, M_{\theta x}$ derived under this assumption are

$$
\begin{aligned}
N_{x}= & \frac{E h}{1-\nu^{2}}\left(\varepsilon_{x}+\nu \varepsilon_{\theta}\right)+\sum_{i=1}^{s} \frac{2 E_{p e} h_{p e}}{1-\nu_{p e}^{2}}\left(\varepsilon_{x}+\nu_{p e} \varepsilon_{\theta}\right) \chi_{p e_{i}}(x, \theta) \\
& +\frac{c_{D} h}{1-\nu^{2}}\left(\dot{\varepsilon}_{x}+\nu \dot{\varepsilon}_{\theta}\right)+\sum_{i=1}^{s} \frac{2 c_{D_{p e}} h_{p e}}{1-\nu_{p e}^{2}}\left(\dot{\varepsilon}_{x}+\nu_{p e} \dot{\varepsilon}_{\theta}\right) \chi_{p e_{i}}(x, \theta) \\
N_{x \theta}= & N_{\theta x}=\frac{E h}{2(1+\nu)} \varepsilon_{x \theta}+\sum_{i=1}^{s} \frac{E_{p e} h_{p e}}{\left(1+\nu_{p e}\right)} \varepsilon_{x \theta} \chi_{p e_{i}}(x, \theta) \\
& +\frac{c_{D} h}{2(1+\nu)} \dot{\varepsilon}_{x \theta}+\sum_{i=1}^{s} \frac{c_{D_{p e}} h_{p e}}{\left(1+\nu_{p e}\right)} \dot{\varepsilon}_{x \theta} \chi_{p e_{i}}(x, \theta)
\end{aligned}
$$


and

$$
\begin{aligned}
M_{x}= & \frac{E h^{3}}{12\left(1-\nu^{2}\right)}\left(\kappa_{x}+\nu \kappa_{\theta}\right)+\sum_{i=1}^{s} \frac{2 E_{p e} a_{3}}{3\left(1-\nu_{p e}^{2}\right)}\left(\kappa_{x}+\nu_{p e} \kappa_{\theta}\right) \chi_{p e_{i}}(x, \theta) \\
& +\frac{c_{D} h^{3}}{12\left(1-\nu^{2}\right)}\left(\dot{\kappa}_{x}+\nu \dot{\kappa}_{\theta}\right)+\sum_{i=1}^{s} \frac{2 c_{D_{p e}} a_{3}}{3\left(1-\nu_{p e}^{2}\right)}\left(\dot{\kappa}_{x}+\nu_{p e} \dot{\kappa}_{\theta}\right) \chi_{p e_{i}}(x, \theta) \\
M_{x \theta}= & M_{\theta x}=\frac{E h^{3}}{24(1+\nu)} \tau+\sum_{i=1}^{s} \frac{E_{p e} a_{3}}{3\left(1+\nu_{p e}\right)} \tau \chi_{p e_{i}}(x, \theta) \\
& \quad+\frac{c_{D} h^{3}}{24(1+\nu)} \dot{\tau}+\sum_{i=1}^{s} \frac{c_{D_{p e}} a_{3}}{3\left(1+\nu_{p e}\right)} \dot{\tau} \chi_{p e_{i}}(x, \theta)
\end{aligned}
$$

The constant $a_{3} \equiv\left(h / 2+h_{p e}\right)^{3}-h^{3} / 8$ results from integration through the thickness of the patch while the characteristic function

$$
\chi_{p e_{i}}(x, \theta)= \begin{cases}1, & x_{1 i} \leq x \leq x_{2 i}, \theta_{1 i} \leq \theta \leq \theta_{2 i} \\ 0, & \text { otherwise }\end{cases}
$$

delineates the region covered by the $i^{\text {th }}$ patch pair with edges at $x_{1 i}, x_{2 i}, \theta_{1 i}, \theta_{2 i}$. Expressions for the resultants $N_{\theta}$ and $M_{\theta}$ can be obtained by replacing $\varepsilon_{x}, \varepsilon_{\theta}, \kappa_{x}, \kappa_{\theta}$ in the expressions for $N_{x}$ and $M_{x}$ by $\varepsilon_{\theta}, \varepsilon_{x}, \kappa_{\theta}, \kappa_{x}$, respectively. As detailed in [8, 10, 21], the midsurface strains and changes in curvature for the Donnell-Mushtari model are

$$
\begin{array}{llll}
\varepsilon_{x}=\frac{\partial u}{\partial x}, & \varepsilon_{\theta}=\frac{1}{R} \frac{\partial v}{\partial \theta}+\frac{w}{R}, & \varepsilon_{x \theta}=\frac{\partial v}{\partial x}+\frac{1}{R} \frac{\partial u}{\partial \theta} \\
\kappa_{x}=-\frac{\partial^{2} w}{\partial x^{2}} \quad, & \kappa_{\theta}=-\frac{1}{R^{2}} \frac{\partial^{2} w}{\partial \theta^{2}} \quad, & \tau=-\frac{2}{R} \frac{\partial^{2} w}{\partial x \partial \theta} .
\end{array}
$$

Note that for the undamped shell which is devoid of patches, substitution of the strain and curvature expressions (4) into the resultant equations (2) and (3) yields the classical DonnellMushtari expressions

$$
\begin{array}{ll}
N_{x}=\frac{E h}{\left(1-\nu^{2}\right)}\left[\frac{\partial u}{\partial x}+\frac{\nu}{R}\left(\frac{\partial v}{\partial \theta}+w\right)\right], & M_{x}=\frac{-E h^{3}}{12\left(1-\nu^{2}\right)}\left[\frac{\partial^{2} w}{\partial x^{2}}+\frac{\nu}{R^{2}} \frac{\partial^{2} w}{\partial \theta^{2}}\right] \\
N_{\theta}=\frac{E h}{\left(1-\nu^{2}\right)}\left[\frac{1}{R} \frac{\partial v}{\partial \theta}+\frac{w}{R}+\nu \frac{\partial u}{\partial x}\right], & M_{\theta}=\frac{-E h^{3}}{12\left(1-\nu^{2}\right)}\left[\frac{1}{R^{2}} \frac{\partial^{2} w}{\partial \theta^{2}}+\nu \frac{\partial^{2} w}{\partial x^{2}}\right] \\
N_{x \theta}=N_{\theta x}=\frac{E h}{2(1+\nu)}\left[\frac{\partial v}{\partial x}+\frac{1}{R} \frac{\partial u}{\partial \theta}\right], & M_{x \theta}=M_{\theta x}=\frac{-E h^{3}}{12 R(1+\nu)} \frac{\partial^{2} w}{\partial x \partial \theta}
\end{array}
$$

(e.g., see [21]).

The necessity of including the passive (material) patch contributions in the density and moment and force resultants for the combined structure is discussed in [10]. While the passive patch contributions may be negligible in some cases, it is well documented that for many currently employed control configurations, the structural dynamics are sufficiently altered by 
the presence of the patches so as to yield inadequate model fits if the patches are neglected. Experimental results demonstrating this effect for circular plates can be found in $[7,10]$ while independent experimental confirmation for rectangular plates is given in [14]. Further analysis for thin shells is provided in [27]. The numerical examples in Section 5 of this paper illustrate that the control method is equally applicable when passive patch contributions are neglected or included and hence is effective when their passive contribution is significant.

To characterize the external or active patch contributions, it is typical to start with the assumption that the strains generated by a patch are proportional to the applied voltage [8]. Since differing voltages can be applied to the outer and inner patches in the pair, we will differentiate between the two with $V_{i 1}(t)$ and $V_{i 2}(t)$ used to denote the voltages to the outer and inner patches in the $i^{\text {th }}$ pair, respectively. The proportionality constant relating the generated strain to the input voltage is designated by $d_{31}$. As detailed in [8], the total external moments and forces generated by the patches are

$$
\begin{aligned}
& \left(M_{x}\right)_{p e_{i}}=\frac{-E_{p e}}{1-\nu_{p e}} \cdot \frac{d_{31}}{h_{p e}} \chi_{p e_{i}}\left[\left(\frac{a_{2}}{2}+\frac{a_{3}}{3 R}\right) V_{i 1}-\left(\frac{a_{2}}{2}-\frac{a_{3}}{3 R}\right) V_{i 2}\right] \\
& \left(M_{\theta}\right)_{p e_{i}}=\frac{-E_{p e}}{1-\nu_{p e}} \cdot \frac{d_{31} a_{2}}{2 h_{p e}} \chi_{p e_{i}}\left[V_{i 1}-V_{i 2}\right] \\
& \left(N_{x}\right)_{p e_{i}}=\frac{-E_{p e}}{1-\nu_{p e}} \cdot \frac{d_{31}}{h_{p e}} \chi_{p \epsilon_{i}} \mathcal{S}_{p e_{i}}\left[\left(h_{p e}+\frac{a_{2}}{2 R}\right) V_{i 1}+\left(h_{p e}-\frac{a_{2}}{2 R}\right) V_{i 2}\right] \\
& \left(N_{\theta}\right)_{p \epsilon_{i}}=\frac{-E_{p e}}{1-\nu_{p e}} d_{31} \chi_{p e_{i}} \mathcal{S}_{p e_{i}}\left[V_{i 1}+V_{i 2}\right]
\end{aligned}
$$

where $a_{2}=\left(h / 2+h_{p e}\right)^{2}-h^{2} / 4$ and $a_{3}=\left(h / 2+h_{p e}\right)^{3}-h^{3} / 8$. When substituted into (1), the expressions (5) provide the input from the patches when voltages are applied. We point out that the characteristic functions $\chi_{p e_{i}}$ restrict the external patch resultants to the region covered by the $i^{\text {th }}$ patch pair.

The fixed-edge boundary conditions

$$
u=v=w=\frac{d w}{\partial x}=0 \quad, \quad x=0, \ell
$$

are used to model the end behavior of the shell. These boundary conditions are appropriate for experimental setups in which heavy endcaps prevent edge movement. Note that alternative boundary conditions such as simply supported or "almost fixed" (see [9]) can be employed if edge movement is suspected.

We consider two sets of initial conditions. For general systems, the initial conditions are specified as

$$
u(0, x)=u_{0}(x) \quad, \quad v(0, x)=v_{0}(x) \quad, \quad w(0, x)=w_{0}(x) .
$$

To model the long-term dynamics of systems driven by a periodic exogenous force $g$ with period $\tau$, we also consider the periodic conditions

$$
u(0, x)=u(\tau, x) \quad, \quad v(0, x)=v(\tau, x) \quad, \quad w(0, x)=w(\tau, x)
$$




\subsection{Weak Form of Modeling Equations}

The internal and external moment and force resultants (2), (3) and (5) are discontinuous due to the presence of the piezoceramic patches. When incorporated in the strong form of the shell model (1), this leads to problems associated with the differentiation of Dirac distributions. To alleviate these difficulties and reduce smoothness requirements on solutions, it is advantageous to consider a weak form of the modeling equations which can be derived from Hamilton's principle (energy considerations).

The state variables for the problem in second-order form are taken to be $y=(u, v, w)$ in the state space $H=L^{2}\left(\Gamma_{0}\right) \times L^{2}\left(\Gamma_{0}\right) \times L^{2}\left(\Gamma_{0}\right)$. For the fixed-edge boundary conditions (6), the space of test functions is taken to be $V=H_{0}^{1}\left(\Gamma_{0}\right) \times H_{0}^{1}\left(\Gamma_{0}\right) \times H_{0}^{2}\left(\Gamma_{0}\right)$ where

$$
\begin{aligned}
& H_{0}^{1}\left(\Gamma_{0}\right)=\left\{\eta \in H^{1}\left(\Gamma_{0}\right) \mid \eta(0, \theta)=\eta(\ell, \theta)=0\right\} \\
& H_{0}^{2}\left(\Gamma_{0}\right)=\left\{\eta \in H^{2}\left(\Gamma_{0}\right) \mid \eta(0, \theta)=\eta_{x}(0, \theta)=\eta(\ell, \theta)=\eta_{x}(\ell, \theta)=0\right\} .
\end{aligned}
$$

For $\Phi=(u, v, w)$ and $\Psi=\left(\eta_{1}, \eta_{2}, \eta_{3}\right)$, the $H$ and $V$ inner products are taken to be

$$
\langle\Phi, \Psi\rangle_{H}=\int_{\Gamma_{0}} \rho h u \bar{\eta}_{1} d \gamma+\int_{\Gamma_{0}} \rho h v \bar{\eta}_{2} d \gamma+\int_{\Gamma_{0}} \rho h w \bar{\eta}_{3} d \gamma
$$

and

$$
\begin{aligned}
& \left\langle\left(E, E_{p \epsilon}\right) \Phi, \Psi\right\rangle_{V}=\int_{\Gamma_{0}}\left\{\frac{E h}{1-\nu^{2}}\left[\left(\varepsilon_{x}+\nu \varepsilon_{\theta}\right) \overline{\frac{\partial \eta_{1}}{\partial x}}+\frac{1}{2 R}(1-\nu) \varepsilon_{x \theta} \frac{\overline{\partial \eta_{1}}}{\partial \theta}\right]\right. \\
& \left.+\sum_{i=1}^{s} \frac{2 E_{p e} h_{p e}}{1-\nu_{p e}^{2}} \chi_{p \epsilon_{i}}(x, \theta)\left[\left(\varepsilon_{x}+\nu_{p e} \varepsilon_{\theta}\right) \frac{\overline{\partial \eta_{1}}}{\partial x}+\frac{1}{2 R}\left(1-\nu_{p e}\right) \varepsilon_{x \theta} \overline{\frac{\partial \eta_{1}}{\partial \theta}}\right]\right\} d \gamma \\
& +\int_{\Gamma_{0}}\left\{\frac{E h}{1-\nu^{2}}\left[\left(\varepsilon_{\theta}+\nu \varepsilon_{x}\right) \overline{\frac{\partial \eta_{1}}{\partial \theta}}+\frac{1}{2 R}(1-\nu) \varepsilon_{x \theta} \overline{\frac{\partial \eta_{2}}{\partial x}}\right]\right. \\
& \left.+\sum_{i=1}^{s} \frac{2 E_{p e} h_{p e}}{1-\nu_{p e}^{2}} \chi_{p \epsilon_{i}}(x, \theta)\left[\left(\varepsilon_{\theta}+\nu_{p e} \varepsilon_{x}\right) \frac{\overline{\partial \eta_{2}}}{\partial \theta}+\frac{1}{2 R}\left(1-\nu_{p e}\right) \varepsilon_{x \theta} \overline{\frac{\partial \eta_{2}}{\partial x}}\right]\right\} d \gamma \\
& +\int_{\Gamma_{0}}\left\{\frac { E h } { 1 - \nu ^ { 2 } } \left[\frac{1}{R}\left(\varepsilon_{\theta}+\nu \varepsilon_{x}\right) \overline{\eta_{3}}-\frac{h^{2}}{12}\left(\kappa_{x}+\nu \kappa_{\theta}\right) \frac{\overline{\partial^{2} \eta_{3}}}{\partial x^{2}}\right.\right. \\
& \left.-\frac{h^{2}}{12 R^{2}}\left(\kappa_{\theta}+\nu \kappa_{x}\right) \overline{\frac{\partial^{2} \eta_{3}}{\partial \theta^{2}}}-\frac{h^{2}}{12 R}(1-\nu) \tau \overline{\frac{\partial^{2} \eta_{3}}{\partial x \partial \theta}}\right] \\
& +\sum_{i=1}^{s} \frac{2 E_{p e}}{3\left(1-\nu_{p e}^{2}\right)} \chi_{p e_{i}}(x, \theta)\left[\frac{3 h_{p e}}{R}\left(\varepsilon_{\theta}+\nu_{p e} \varepsilon_{x}\right) \overline{\eta_{3}}-a_{3}\left(\kappa_{x}+\nu_{p e} \kappa_{\theta}\right) \frac{\overline{\partial^{2} \eta_{3}}}{\partial x^{2}}\right. \\
& \left.\left.-\frac{a_{3}}{R^{2}}\left(\kappa_{\theta}+\nu_{p e} \kappa_{x}\right) \overline{\frac{\partial^{2} \eta_{3}}{\partial \theta^{2}}}-\frac{a_{3}}{R}\left(1-\nu_{p e}\right) \tau \overline{\frac{\partial^{2} \eta_{3}}{\partial x \partial \theta}}\right]\right\} d \gamma
\end{aligned}
$$

where $\varepsilon_{x}, \varepsilon_{\theta}, \varepsilon_{x \theta}, \kappa_{x}, \kappa_{\theta}, \tau$ are defined in (4) and $d \gamma=R d \theta d x$. The dependence of the inner product on the Young's moduli is explicitly included in the definition to provide a notation for defining analogous damping expressions later in this work. 
The weak form of (1), as derived in [10] from energy principles, is given by

$$
\begin{gathered}
\int_{\Gamma_{0}}\left\{R \rho h \frac{\partial^{2} u}{\partial t^{2}} \bar{\eta}_{1}+R N_{x} \frac{\overline{\partial \eta_{1}}}{\partial x}+N_{\theta x} \frac{\overline{\partial \eta_{1}}}{\partial \theta}-R \hat{q}_{x} \bar{\eta}_{1}-R \sum_{i=1}^{s}\left(N_{x}\right)_{p e_{i}} \overline{\frac{\partial \eta_{1}}{\partial x}}\right\} d \gamma=0 \\
\int_{\Gamma_{0}}\left\{R \rho h \frac{\partial^{2} v}{\partial t^{2}} \bar{\eta}_{2}+N_{\theta} \frac{\overline{\partial \eta_{2}}}{\partial \theta}+R N_{x \theta} \frac{\overline{\partial \eta_{2}}}{\partial x}-R \hat{q}_{\theta} \bar{\eta}_{2}-\sum_{i=1}^{s}\left(N_{\theta}\right)_{p e_{i}} \frac{\overline{\partial \eta_{2}}}{\partial \theta}\right\} d \gamma=0 \\
\int_{\Gamma_{0}}\left\{R \rho h \frac{\partial^{2} w}{\partial t^{2}} \bar{\eta}_{3}+R \mu \frac{\partial w}{\partial t} \bar{\eta}_{3}+N_{\theta} \bar{\eta}_{3}-R M_{x} \frac{\overline{\partial^{2} \eta_{3}}}{\partial x^{2}}-\frac{1}{R} M_{\theta} \frac{\overline{\partial^{2} \eta_{3}}}{\partial \theta^{2}}-2 M_{x \theta} \frac{\overline{\partial^{2} \eta_{3}}}{\partial x \partial \theta}\right. \\
\left.-R \hat{q}_{n} \bar{\eta}_{3}+\sum_{i=1}^{s}\left[R\left(M_{x}\right)_{p e_{i}} \frac{\overline{\partial^{2} \eta_{3}}}{\partial x^{2}}+\frac{1}{R}\left(M_{\theta}\right)_{p e_{i}} \frac{\overline{\partial^{2} \eta_{3}}}{\partial \theta^{2}}\right]\right\} d \gamma=0
\end{gathered}
$$

for all $\Psi=\left(\eta_{1}, \eta_{2}, \eta_{3}\right) \in V$. A comparison between (7) and (1) illustrates that in the weak form, derivatives are transferred from the discontinuous resultants onto suitably smooth test functions. The problem in this form is then amenable to analysis and approximation.

\subsection{Abstract Formulation}

To provide a natural framework in which to establish model well-posedness and infinite dimensional LQR control methods, we consider an abstract formulation of the model based upon stiffness and damping sesquilinear forms. To this end, we define $\sigma_{i}: V \times V \rightarrow \mathbb{C}, i=1,2$ by

$$
\begin{aligned}
& \sigma_{1}(\Phi, \Psi)=\left\langle\left(E, E_{p e}\right) \Phi, \Psi\right\rangle_{V} \\
& \sigma_{2}(\Phi, \Psi)=\left\langle\left(c_{D}, c_{D_{p e}}\right) \Phi, \Psi\right\rangle_{V}+\int_{\Gamma_{0}} \mu w \bar{\eta}_{3} d \gamma
\end{aligned}
$$

Note that $\left\langle\left(c_{D}, c_{D_{p e}}\right) \Phi, \Psi\right\rangle_{V}$ differs from $\left\langle\left(E, E_{p e}\right) \Phi, \Psi\right\rangle_{V}$ only in that Young's moduli are replaced by Kelvin-Voigt damping coefficients. It can be directly verified that the stiffness form $\sigma_{1}$ satisfies

$$
\begin{array}{ll}
\left|\sigma_{1}(\Phi, \Psi)\right| \leq c_{1}|\Phi|_{V}|\Psi|_{V}, \text { for some } c_{1} \in \mathbb{R} & \text { (Bounded) } \\
\operatorname{Re} \sigma_{1}(\Phi, \Phi) \geq c_{2}|\Phi|_{V}^{2}, \text { for some } c_{2}>0 & (V \text {-Elliptic) } \\
\sigma_{1}(\Phi, \Psi)=\overline{\sigma_{1}(\Psi, \Phi)} & \text { (Symmetric) }
\end{array}
$$

for all $\Phi, \Psi \in V$. Moreover, the damping term $\sigma_{2}$ satisfies

$$
\begin{array}{ll}
\left|\sigma_{2}(\Phi, \Psi)\right| \leq c_{3}|\Phi|_{V}|\Psi|_{V}, \text { for some } c_{3} \in \mathbb{R} & \text { (Bounded) } \\
\operatorname{Re} \sigma_{2}(\Phi, \Phi) \geq c_{4}|\Phi|_{V}^{2}, \text { for some } c_{4}>0 & \text { (V-Elliptic) }
\end{array}
$$


Remark 1. The symmetry of $\sigma_{1}$ is dependent upon the choice of shell model and ultimately reflects the Maxwell-Betti reciprocity theorem. While the Donnell-Mushtari model yields a symmetric sesquilinear form $\sigma_{1}$, other models such as the Timoshenko shell model will not yield a symmetric form.

The control input space is taken to be the Hilbert space $U=\mathbb{R}^{2 s}$ and for $\Psi \in V$, the input operator $B \in \mathcal{L}\left(U, V^{*}\right)$ is defined by

$$
\begin{array}{r}
\langle B u(t), \Psi\rangle_{V^{*}, V}=\int_{\Gamma_{0}} \sum_{i=1}^{s}\left\{\left(N_{x}\right)_{p \epsilon_{i}} \frac{\overline{\partial \eta_{1}}}{\partial x}+\frac{1}{R}\left(N_{\theta}\right)_{p \epsilon_{i}} \frac{\overline{\partial \eta_{2}}}{\partial \theta}\right. \\
\left.\quad-\left(M_{x}\right)_{p e_{i}} \frac{\overline{\partial^{2} \eta_{3}}}{\partial x^{2}}-\frac{1}{R^{2}}\left(M_{\theta}\right)_{p e_{i}} \frac{\overline{\partial^{2} \eta_{3}}}{\partial \theta^{2}}\right\} d \gamma
\end{array}
$$

where $\langle\cdot, \cdot\rangle_{V^{*}, V}$ denotes the usual duality product. With the definition $\tilde{g}=(1 / \rho h)\left[\hat{q}_{x}, \hat{q}_{\theta}, \hat{q}_{n}\right]$, we can write the weak form (7) in the abstract variational form

$$
\langle\ddot{y}(t), \Psi\rangle_{V^{*}, V}+\sigma_{2}(\dot{y}(t), \Psi)+\sigma_{1}(y(t), \Psi)=\langle B u(t)+\tilde{g}(t), \Psi\rangle_{V^{*}, V} .
$$

To pose the problem in a first-order form amenable for control applications, we define the product spaces $\mathcal{H}=V \times H$ and $\mathcal{V}=V \times V$ with the norms

$$
\begin{aligned}
\left|\left(\phi_{1}, \phi_{2}\right)\right|_{\mathcal{H}}^{2} & =\left|\phi_{1}\right|_{V}^{2}+\left|\phi_{2}\right|_{H}^{2} \\
\left|\left(\phi_{1}, \phi_{2}\right)\right|_{\mathcal{V}}^{2} & =\left|\phi_{1}\right|_{V}^{2}+\left|\phi_{2}\right|_{V}^{2} .
\end{aligned}
$$

The state is taken to be $z(t)=(y(t), \dot{y}(t)) \in \mathcal{H}$ while the product space forcing terms are formulated as

$$
g(t)=\left[\begin{array}{c}
0 \\
g(t)
\end{array}\right] \quad, \quad \mathcal{B} u(t)=\left[\begin{array}{c}
0 \\
B u(t)
\end{array}\right] .
$$

The second-order system (9) can then be formulated in the first-order form

$$
\begin{aligned}
& \langle\dot{z}(t), \Lambda\rangle_{\mathcal{L}, \mathcal{V}}+\sigma(z(t), \Lambda)=\langle\mathcal{B} u(t)+g(t), \Lambda\rangle_{\mathcal{V} * \mathcal{V}} \quad \text { for } \Lambda \in \mathcal{V} \\
& z(0)=z_{0}=\left(y_{0}, y_{1}\right)
\end{aligned}
$$

where $\sigma: \mathcal{V} \times \mathcal{V} \rightarrow \mathbb{C}$ is given by

$$
\sigma(\phi, \psi)=-\left\langle\phi_{2}, \psi_{1}\right\rangle_{V}+\sigma_{1}\left(\phi_{1}, \psi_{2}\right)+\sigma_{2}\left(\phi_{2}, \psi_{2}\right)
$$

for $\phi=\left(\phi_{1}, \phi_{2}\right), \psi=\left(\psi_{1}, \psi_{2}\right) \in \mathcal{V}$. As proven in [10, page 109], $\sigma$ is $\mathcal{V}$ continuous and for $\lambda>0, \sigma(\cdot, \cdot)+\lambda\langle\cdot, \cdot\rangle_{\mathcal{H}}$ is $\mathcal{V}$-elliptic. From the continuity of $\sigma$, it follows that one can define an operator $\tilde{\mathcal{A}} \in \mathcal{L}\left(\mathcal{V}, \mathcal{V}^{*}\right)$ by $\sigma(\Upsilon, \Lambda)=\langle\tilde{\mathcal{A}} \Upsilon, \Lambda\rangle_{\mathcal{V}^{*}, \mathcal{V}}$.

To obtain a strong form of the first-order system (11), consider the system operator

$$
\begin{aligned}
& \operatorname{dom} \mathcal{A}=\left\{\left(\phi_{1}, \phi_{2}\right) \in \mathcal{H} \mid \phi_{2} \in V, A_{1} \phi_{1}+A_{2} \phi_{2} \in H\right\} \\
& \mathcal{A}=\left[\begin{array}{cc}
0 & I \\
-A_{1} & -A_{2}
\end{array}\right]
\end{aligned}
$$


with $A_{1}, A_{2} \in \mathcal{L}\left(V, V^{*}\right)$ defined by

$$
\left\langle A_{i} \phi_{1}, \phi_{2}\right\rangle_{V^{*}, V}=\sigma_{i}\left(\phi_{1}, \phi_{2}\right) \quad, \quad i=1,2
$$

It should be notated that $\mathcal{A}$ is the negative of the restriction to $\operatorname{dom} \mathcal{A}$ of $\tilde{\mathcal{A}} \in \mathcal{L}\left(\mathcal{V}, \mathcal{V}^{*}\right)$ so that $\sigma(\Upsilon, \Lambda)=\langle-\mathcal{A} \Upsilon, \Lambda\rangle_{\mathcal{H}}$ for $\Upsilon \in \operatorname{dom} \mathcal{A}, \Lambda \in \mathcal{V}$. A strong form of the abstract system model is then given by

$$
\begin{aligned}
& \dot{z}(t)=\mathcal{A} z(t)+\mathcal{B} u(t)+g(t) \text { in } \mathcal{V}^{*}=V \times V^{*} \\
& z(0)=z_{0} .
\end{aligned}
$$

The rigorous equivalence of solutions is established through the following theorems.

Theorem 1. Under Hypotheses (H1)-(H5) on $\sigma_{1}$ and $\sigma_{2}$, $\widetilde{\mathcal{A}}$ generates an analytic semigroup $\mathcal{T}(t)$ on $\mathcal{V}, \mathcal{H}$ and $\mathcal{V}^{*}$. In terms of this semigroup, the representation

$$
z(t)=\mathcal{T}(t) z_{0}+\int_{0}^{t} \mathcal{T}(t-s)[\mathcal{B} u(s)+g(s)] d s
$$

defines a mild solution to (13) for $z_{0} \in \mathcal{V}^{*}$ and $\mathcal{B} u+g \in L^{2}\left((0, T) ; \mathcal{V}^{*}\right)$. Furthermore, this semigroup is (uniformly) exponentially stable on $\mathcal{V}, \mathcal{H}$ and $\mathcal{V}^{*}$.

Theorem 2. Let $z_{s g}$ denote the semigroup solution to (13) given by (14) and let $z_{\text {var }}$ denote the weak solution to $(9)$. Under hypotheses (H1)-(H5), it follows that $z_{s g}\left(z_{0}, \mathcal{F}\right)=z_{\text {var }}\left(z_{0}, \mathcal{F}\right)$ for $z_{0} \in \mathcal{H}$ and $\mathcal{F} \equiv \mathcal{B} u+g \in L^{2}\left((0, T) ; \mathcal{V}^{*}\right)$.

Following the convention of [28], we will use the same notation for the semigroups defined on $\mathcal{V}, \mathcal{H}$ and $\mathcal{V}^{*}$ since each semigroup is an extension or restriction of the others. Note that $\operatorname{dom} \mathcal{A}$ defined in $(12)$ is actually $\operatorname{dom}_{\mathcal{H}} \widetilde{\mathcal{A}}$, the domain of $\widetilde{\mathcal{A}}$ as a generator of $\mathcal{T}(t)$ in $\mathcal{H}$. As detailed in Lemma 3.6.1 and Theorem 3.6.1 of [28] (see also Section IV.6 of [24] and Chapter 2, Theorem 5.2 of [23]), the property that $\tilde{\mathcal{A}}$ generates an analytic semigroup on $\mathcal{V}, \mathcal{H}$ and $\mathcal{V}^{*}$ results from the continuity and $\mathcal{V}$-ellipticity of $\sigma$. The exponential stability of $\mathcal{T}(t)$ on $\mathcal{H}$ for second-order systems with strong damping is demonstrated in [4] while the exponential stability of $\mathcal{T}(t)$ on $\mathcal{V}$ and $\mathcal{V}^{*}$ in this setting is proven in Lemma 3.3 of [5]. Finally, Theorem 2 is a reformulation of Theorem 4.14 of [10] and details can be found therein.

Remark 2. The previous analysis applies to general $\mathcal{V}^{*}$-valued forces with states having initial values $z_{0} \in \mathcal{V}^{*}$. In many applications, disturbances are generated by rotating or oscillating components in which case it is reasonable to assume that $g$ is periodic. The long-term behavior of the system then satisfies the abstract Cauchy equation

$$
\begin{aligned}
& \dot{z}(t)=\mathcal{A} z(t)+\mathcal{B} u(t)+g(t) \\
& z(0)=z(\tau) .
\end{aligned}
$$

in $\mathcal{V}^{*}$ where $\tau$ is taken to be commensurate with all frequencies present in the disturbance $g$. 


\section{Approximation Problem}

For implementation purposes, we consider Galerkin approximations to obtain trajectories which evolve in finite dimensional subspaces $\mathcal{V}^{N} \subset \mathcal{V} \subset \mathcal{H}$. To guarantee convergence in the subsequent control problem, we assume that the approximation method satisfies the standard convergence condition

$(\mathrm{H} 1 \mathrm{~N})$ For any $z \in \mathcal{V}$, there exists a sequence $\tilde{z}^{N} \in \mathcal{V}^{N}$ such that $\left|z-\tilde{z}^{N}\right| \mathcal{V} \rightarrow 0$ as $N \rightarrow \infty$

In this framework, the operator $\mathcal{A}^{N}: \mathcal{V}^{N} \rightarrow \mathcal{V}^{N}$ which approximates $\mathcal{A}$ is defined by restricting $\sigma$ to $\mathcal{V}^{N} \times \mathcal{V}^{N}$ to yield

$$
\left\langle-\mathcal{A}^{N} \Upsilon, \Lambda\right\rangle_{\mathcal{H}}=\sigma(\Upsilon, \Lambda) \quad \text { for all } \Upsilon, \Lambda \in \mathcal{V}^{N}
$$

For each $N$, the $C_{0}$ semigroup on $\mathcal{V}^{N}$ which is generated by $\mathcal{A}^{N}$ is denoted by $\mathcal{T}^{N}(t)$. The control operator is approximated by $\mathcal{B}^{N} \in \mathcal{L}\left(U, \mathcal{V}^{N}\right)$ given by

$$
\left\langle\mathcal{B}^{N} u, \Lambda\right\rangle_{\mathcal{H}}=\left\langle u, \mathcal{B}^{*} \Lambda\right\rangle_{U} \quad \text { for all } u \in U, \Lambda \in \mathcal{V}^{N}
$$

Finally, we let $\mathcal{P}^{N}$ denote the usual orthogonal projection of $\mathcal{H}$ onto $\mathcal{V}^{N}$ which by definition satisfies

(i) $\mathcal{P}^{N} \Upsilon \in \mathcal{V}^{N} \quad$ for $\Upsilon \in \mathcal{H}$

(ii) $\left\langle\mathcal{P}^{N} \Upsilon-\Upsilon, \Lambda\right\rangle_{\mathcal{H}}=0$ for all $\Lambda \in \mathcal{V}^{N}$.

This projection can be extended to $\mathcal{P}^{N} \in \mathcal{L}\left(\mathcal{V}^{*}, \mathcal{V}^{N}\right)$ by replacing the $\mathcal{H}$-inner product $\langle\Upsilon, \Lambda\rangle_{\mathcal{H}}$ by the duality product $\langle\Upsilon, \Lambda\rangle_{\mathcal{V}^{*}, \mathcal{V}}$ and considering $\Upsilon \in \mathcal{V}^{*}$.

The approximate problem corresponding to (11) can then be formulated as

$$
\begin{aligned}
& \left\langle\dot{z}^{N}(t), \Lambda\right\rangle_{\mathcal{H}}+\sigma\left(z^{N}(t), \Lambda\right)=\left\langle\mathcal{B}^{N} u(t)+\mathcal{P}^{N} g(t), \Lambda\right\rangle_{\mathcal{H}} \quad \text { for all } \Lambda \in \mathcal{V}^{N} \\
& z^{N}(0)=\mathcal{P}^{N} z_{0} .
\end{aligned}
$$

with the solution given by

$$
z^{N}(t)=\mathcal{T}^{N}(t) \mathcal{P}^{N} z_{0}+\int_{0}^{t} \mathcal{T}^{N}(t-s)\left[\mathcal{B}^{N} u(s)+\mathcal{P}^{N} g(s)\right] d s
$$

In strong form, the finite dimensional system has the form

$$
\begin{aligned}
& \dot{z}^{N}(t)=\mathcal{A}^{N} z^{N}(t)+\mathcal{B}^{N} u(t)+\mathcal{P}^{N} g(t) \\
& z^{N}(0)=\mathcal{P}^{N} z_{0}
\end{aligned}
$$

This system forms the constraint equations used in Section 4.1. A similar finite dimensional system approximates the dynamics of the periodic system.

The exponential stability of the semigroup $\mathcal{T}(t)$ was established in Theorem 1 . An important issue in the subsequent control formulation concerns the uniform stability of the semigroup under approximation and this is established in the following theorem from [5, 10] 
(see specifically Lemma 7.13 of [10]). The use of the theorem for our shell system is illustrated in Example 1 of the next section.

Theorem 3. Assume that the injection $i: V \hookrightarrow H$ is compact. It is also assumed that the damping sesquilinear form can be decomposed as $\sigma_{2}=\delta \sigma_{1}+\hat{\sigma}_{2}$, for some $\delta>0$, where the continuous sesquilinear form $\hat{\sigma}_{2}$ satisfies for some $\lambda \in \mathbb{R}$

$$
\operatorname{Re} \hat{\sigma}_{2}(\phi, \phi) \geq-\frac{\delta}{2}|\phi|_{V}^{2}-\lambda|\phi|_{H}^{2} \quad \text { for all } \phi \in V \text {. }
$$

Finally, suppose that the operator $A_{1}^{-1} \hat{A}_{2}$, where $\hat{A}_{2} \in \mathcal{L}\left(V, V^{*}\right)$ is defined by $\left\langle\widehat{A}_{2} \phi, \eta\right\rangle_{V^{*}, V}=$ $\hat{\sigma}_{2}(\phi, \eta)$, is compact on $V$.

If for some $\omega \in \mathbb{R}$ and $M \geq 1, \mathcal{T}(t)$ satisfies

$$
|\mathcal{T}(t)|_{\mathcal{L}(\mathcal{H})} \leq M e^{\omega t} \quad, \quad t \geq 0
$$

then for any $\varepsilon>0$ there exists an integer $N_{\varepsilon}$ such that for $N \geq N_{\varepsilon}$,

$$
\left|\mathcal{T}^{N}(t) \mathcal{P}^{N}\right|_{\mathcal{L}(\mathcal{H})} \leq \widetilde{M} e^{(\omega+\varepsilon) t} \quad, \quad t \geq 0
$$

for some constant $\widetilde{M}>0$ independent of $N$.

To obtain matrix representations for the finite-dimensional operators $\mathcal{A}^{N}, \mathcal{B}^{N}$ and $g^{N}$, it is necessary to specify a basis for the approximating subspace $\mathcal{V}^{N}$. To exploit the tensor nature of the shell domain $\Gamma_{0}$ and circumferential periodicity, we employ the basis described in [12] which is constructed with Fourier components in $\theta$ and cubic splines in $x$. Specifically, the component bases for the $u, v$ and $w$ displacements are assumed to have the form $\mathcal{B}_{u_{k}}(\theta, x)=e^{i m \theta} B_{u_{j}}(x), \mathcal{B}_{v_{k}}(\theta, x)=e^{i m \theta} B_{v_{j}}(x)$, and $\mathcal{B}_{w_{k}}(\theta, x)=e^{i m \theta} B_{w_{j}}(x)$ where $B_{u_{j}}, B_{v_{j}}$ and $B_{w_{j}}$ are cubic $B$ splines modified to satisfy the boundary conditions. The approximating subspace then has the form $\mathcal{V}^{N}=\operatorname{span}\left\{\mathcal{B}_{u_{k}}\right\} \times \operatorname{span}\left\{\mathcal{B}_{v_{k}}\right\} \times \operatorname{span}\left\{\mathcal{B}_{w_{k}}\right\}$ and displacements are approximated through the expansions

$$
\begin{aligned}
u^{N}(t, \theta, x) & =\sum_{k=1}^{\mathcal{N}_{u}} u_{k}(t) \mathcal{B}_{u_{k}}(\theta, x) \\
v^{N}(t, \theta, x) & =\sum_{k=1}^{\mathcal{N}_{v}} v_{k}(t) \mathcal{B}_{v_{k}}(\theta, x) \\
w^{N}(t, \theta, x) & =\sum_{k=1}^{\mathcal{N}_{w}} w_{k}(t) \mathcal{B}_{w_{k}}(\theta, x) .
\end{aligned}
$$

The restriction of $\sigma$ to $\mathcal{V}^{N}$ and construction of the forcing vectors then yields the matrix system

$$
\begin{aligned}
& {\left[\begin{array}{cc}
K_{E}^{\mathcal{N}} & 0 \\
0 & M^{\mathcal{N}}
\end{array}\right]\left[\begin{array}{l}
\dot{\vartheta}^{\mathcal{N}}(t) \\
\ddot{\vartheta}^{\mathcal{N}}(t)
\end{array}\right]=\left[\begin{array}{cc}
0 & K_{E}^{\mathcal{N}} \\
-K_{E}^{\mathcal{N}} & -K_{c_{D}}^{\mathcal{N}}
\end{array}\right]\left[\begin{array}{c}
\vartheta^{\mathcal{N}}(t) \\
\dot{\vartheta}^{\mathcal{N}}(t)
\end{array}\right]+\left[\begin{array}{c}
0 \\
\tilde{B}^{\mathcal{N}}
\end{array}\right][u(t)]+\left[\begin{array}{c}
0 \\
\tilde{g}^{\mathcal{N}}(t)
\end{array}\right]} \\
& {\left[\begin{array}{cc}
K_{E}^{\mathcal{N}} & 0 \\
0 & M^{\mathcal{N}}
\end{array}\right]\left[\begin{array}{l}
\vartheta^{\mathcal{N}}(0) \\
\dot{\vartheta}^{\mathcal{N}}(0)
\end{array}\right]=\left[\begin{array}{c}
z_{1}^{\mathcal{N}} \\
z_{2}^{\mathcal{N}}
\end{array}\right]}
\end{aligned}
$$


where the vector $\vartheta^{\mathcal{N}}(t)=\left[u_{1}(t), \cdots, u_{\mathcal{N}_{u}}(t), v_{1}(t), \cdots, v_{\mathcal{N}_{v}}(t), w_{1}(t), \cdots, w_{\mathcal{N}_{w}}(t)\right]^{T}$ contains the $\mathcal{N}=\mathcal{N}_{u}+\mathcal{N}_{v}+\mathcal{N}_{w}$ generalized Fourier coefficients. The $s$ patch inputs are contained in the vector $u(t)=\left[u_{1}(t), \cdots, u_{s}(t)\right]^{T}$. The reader is referred to [12] for details concerning the construction of the mass, stiffness and damping matrices $M^{\mathcal{N}}, K_{E}^{\mathcal{N}}, K_{c_{D}}^{\mathcal{N}}$, the inputs $\tilde{B}^{\mathcal{N}}, \tilde{g}^{\mathcal{N}}(t)$ and the initial conditions $z_{1}^{\mathcal{N}}, z_{2}^{\mathcal{N}}$.

Multiplication by the inverted mass matrix yields the Cauchy equation

$$
\begin{aligned}
& \dot{z}^{N}(t)=A^{N} z^{N}(t)+B^{N} u(t)+g^{N}(t) \\
& z^{N}(0)=z_{0}^{N},
\end{aligned}
$$

where $z^{N}(t)=\left[\vartheta^{\mathcal{N}}(t), \dot{\vartheta}^{\mathcal{N}}(t)\right]^{T} \in \mathbb{R}^{N}$ with $N=2 \mathcal{N}$. Note that $A^{N}$ and $B^{N}$ are the matrix representations for the operators $\mathcal{A}^{N}$ and $\mathcal{B}^{N}$ in terms of the Fourier/spline basis while $g^{N}(t)$ is the projection of $g(t)$. We point out that the notation $z^{N}(t)$ designates both the time-dependent generalized Fourier coefficients in $\mathbb{R}^{N}$ and approximate solutions in $\mathcal{V}^{N}$. The specific usage is indicated by the context and the use of finite dimensional operators or the corresponding matrix representations. Similarly, the control input $u(t)$ should not be confused with the longitudinal displacement $u(t, x, \theta)$. Equation $(20)$ with $g^{N}(t) \equiv 0$ forms the constraint for Example 2 in Section 5.

\section{LQR Control Problem}

As noted in (13) and (15) of the last section, the thin shell model can be posed in the abstract forms

$$
\begin{aligned}
& \dot{z}(t)=\mathcal{A} z(t)+\mathcal{B} u(t)+g(t) \\
& z(0)=z_{0}
\end{aligned}
$$

or

$$
\begin{aligned}
& \dot{z}(t)=\mathcal{A} z(t)+\mathcal{B} u(t)+g(t) \\
& z(0)=z(\tau),
\end{aligned}
$$

in $\mathcal{V}^{*}$, depending upon the periodicity assumptions on the disturbance and state. In this section, infinite dimensional and approximating finite dimensional LQR control methods are developed for the system (21) with $g \equiv 0$ and system (22) with periodic disturbance $g$ and periodic end conditions.

\subsection{No Exogenous Input}

The infinite horizon optimal control problem for systems with no exogenous input $g$ can be posed as follows: determine the control $u$ which minimizes the quadratic cost functional

$$
J\left(u, z_{0}\right)=\int_{0}^{\infty}\left\{|\mathcal{Q} z(t)|_{\mathcal{H}}^{2}+\left|\mathcal{R}^{1 / 2} u(t)\right|_{U}^{2}\right\} d t
$$

subject to

$$
\begin{aligned}
& \dot{z}(t)=\mathcal{A} z(t)+\mathcal{B} u(t) \\
& z(0)=z_{0} .
\end{aligned}
$$


The nonnegative, self-adjoint operator $\mathcal{Q} \equiv \mathcal{D}^{*} \mathcal{D} \in \mathcal{L}(\mathcal{H})$ can be specified to weight state components while the positive, self-adjoint operator $\mathcal{R}=\left(\mathcal{R}^{1 / 2}\right)^{2} \in \mathcal{L}(U)$ serves to weight the control. In this case, $\mathcal{R}$ acts as a soft constraint to prevent excessive voltages to the patches.

To guarantee the existence of a unique Riccati solution and an exponentially stable closed loop semigroup, it is sufficient to require that $(\mathcal{A}, \mathcal{B})$ is stabilizable and $(\mathcal{A}, \mathcal{D})$ is detectable. Under these conditions, it is established in $[5,10]$ that the optimal control minimizing (23) is given by

$$
\bar{u}(t)=-\mathcal{R}^{-1} \mathcal{B}^{*} \Pi \bar{z}(t)
$$

where $\Pi$ solves the algebraic Riccati equation

$$
\left(\mathcal{A}^{*} \Pi+\Pi \mathcal{A}-\Pi \mathcal{B} \mathcal{R}^{-1} \mathcal{B}^{*} \Pi+\mathcal{Q}\right) z=0 \quad \text { for all } z \in \mathcal{V}
$$

and $\bar{z}(t)=\mathcal{S}(t) z_{0}$. Here $\mathcal{S}(t)$ is the closed loop semigroup generated by $\mathcal{A}-\mathcal{B} \mathcal{R}^{-1} \mathcal{B}^{*} \Pi$.

The corresponding finite dimensional problem concerns the determination of suboptimal controls $u$ which minimize the functional

$$
J^{N}\left(u, z_{0}\right)=\int_{0}^{\infty}\left\{\left|\mathcal{Q}^{N} z^{N}(t)\right|_{\mathcal{H}}^{2}+\left|\mathcal{R}^{1 / 2} u(t)\right|_{U}^{2}\right\} d t
$$

subject to

$$
\begin{aligned}
& \dot{z}^{N}(t)=\mathcal{A}^{N} z^{N}(t)+\mathcal{B}^{N} u(t) \\
& z^{N}(0)=\mathcal{P}^{N} z_{0}
\end{aligned}
$$

(see (18) with $g \equiv 0$ ). The following theorem specifies conditions which guarantee the existence and convergence of these suboptimal controls to the control (24) for the original infinite dimensional system. This is Theorem 7.10 of [10]) and details can be found therein. The application of this theorem to the shells system is illustrated in Example 1 which follows.

Theorem 4. Assume that the injection $i: V \hookrightarrow H$ is compact. Let the sesquilinear form $\sigma$ associated with the first-order system (11) be continuous and $\mathcal{V}$-elliptic. Assume that the operators $\mathcal{A}, \mathcal{B}, \mathcal{D}$ satisfy: $(\mathcal{A}, \mathcal{B})$ is stabilizable and $(\mathcal{A}, \mathcal{D})$ is detectable where $\mathcal{B} \in \mathcal{L}\left(U, \mathcal{V}^{*}\right)$ is unbounded. Consider an approximation method which satisfies (H1N). Finally, suppose that for fixed $N_{0}$ and $N>N_{0}$, the pair $\left(\mathcal{A}^{N}, \mathcal{B}^{N}\right)$ is uniformly stabilizable and $\left(\mathcal{A}^{N}, \mathcal{D}^{N}\right)$ is uniformly detectable.

Then for $N$ sufficiently large, there exists a unique nonnegative self-adjoint solution $\Pi^{N} \in$ $\mathcal{L}\left(\mathcal{V}^{*}, \mathcal{V}\right)$ to the $N^{\text {th }}$ approximate algebraic Riccati equation

$$
\mathcal{A}^{N^{*}} \Pi^{N}+\Pi^{N} \mathcal{A}^{N}-\Pi^{N} \mathcal{B}^{N} \mathcal{R}^{-1} \mathcal{B}^{N^{*}} \Pi^{N}+\mathcal{Q}^{N}=0
$$

in $\mathcal{V}^{N}$. There also exist constants $M_{3} \geq 1$ and $\omega_{3}>0$ independent of $N$ such that the closed loop semigroup $\mathcal{S}^{N}(t)=e^{\left(\mathcal{A}^{N}-\mathcal{B}^{N} \mathcal{R}^{-1} \mathcal{B}^{N^{*}} \Pi^{N}\right) t}$ satisfies

$$
\left|\mathcal{S}^{N}(t)\right|_{\mathcal{V}^{N}} \leq M_{3} e^{-\omega_{3} t} \quad, \quad t>0
$$

Moreover, the convergence of the Riccati and control operators

$$
\begin{aligned}
& \Pi^{N} \mathcal{P}^{N} z \stackrel{s}{\rightarrow} \Pi z \text { in } \mathcal{V} \text { for every } z \in \mathcal{V}^{*} \\
& \left|\mathcal{B}^{N^{*}} \Pi^{N} \mathcal{P}^{N}-\mathcal{B}^{*} \Pi\right|_{\mathcal{L}(\mathcal{H}, U)} \rightarrow 0,
\end{aligned}
$$

as $N \rightarrow \infty$, is obtained. 


\section{Example 1.}

We consider in this example a shell with constant parameters $\rho, E, \nu, c_{D}$. Such a case would arise if modeling a homogeneous shell or a shell in which the variance of material properties across regions with actuators is negligible. The sesquilinear forms for this model are specified in (8). Due to the constant coefficients, $\sigma_{2}$ can be written as $\sigma_{2}=\delta \sigma_{1}+\hat{\sigma}_{2}$ where $\delta=\frac{c_{D}}{E}$ and $\hat{\sigma}_{2}(\Phi, \Psi)=\mu \int_{\Gamma_{0}} w \eta_{3} d \gamma$. It follows immediately that

$$
\operatorname{Re} \hat{\sigma}_{2}(\phi, \phi)=\mu \int_{\Gamma_{0}} \phi^{2} d \gamma \geq-\frac{\delta}{2}|\phi|_{V}^{2}
$$

for all $\phi \in V$. The boundedness of the operator $\hat{A}_{2}$ generated by $\hat{\sigma}_{2}$ follows directly from the boundedness of $\hat{\sigma}_{2}$. Furthermore, it is noted that $A_{1}^{-1} \in \mathcal{L}\left(V^{*}, V\right)$ can be written as an operator on $V \rightarrow V$ by $A_{1}^{-1}=A_{1}^{-1} i^{*} i$ where the injections $i: V \hookrightarrow H, i^{*}: H \hookrightarrow V^{*}$ are compact. Thus $A_{1}^{-1}$ is compact on $V$ which implies that $A_{1}^{-1} \hat{A}_{2}$ is compact on $V$ since it is formed from the product of compact and bounded linear operators. Finally, the exponential stability of $\mathcal{T}(t)$, the stabilizability of $(\mathcal{A}, \mathcal{B})$ and the detectability of $(\mathcal{A}, \mathcal{D})$ are guaranteed by Theorem 1. The hypotheses of Theorem 3 are then satisfied for this system and one obtains uniform bounds on the approximating semigroups. The convergence of the Riccati and control operators is then obtained from Theorem 4.

Remark 3. We note that an alternative means of establishing the well-posedness of the closed loop control problem and convergence criteria for suboptimal control gains is through the utilization of uniform analyticity conditions for the semigroup $\mathcal{T}(t)$ generated by $\mathcal{A}$ as discussed in $[16,19]$. For some systems with unbounded input operators, such conditions are more readily verified than the requirements of uniform stabilizability and detectability.

\subsection{Periodic Exogenous Input}

The second case under consideration is that in which the exogenous force $g$ models periodic disturbances such as noise generated by rotating engine components (e.g., propellers or turbines) or periodic electromagnetic sources. Under the assumption that $g$ is periodic in time, the functional to be minimized is

$$
J_{\tau}(u)=\frac{1}{2} \int_{0}^{\tau}\left\{|\mathcal{Q} z(t)|_{\mathcal{H}}^{2}+\left|\mathcal{R}^{1 / 2} u(t)\right|_{U}^{2}\right\} d t
$$

subject to (22). The endpoint $\tau$ is chosen to be commensurate with all frequencies present in $g$. The theory for this case is less complete than that for systems with no exogenous input and is currently limited to bounded control inputs $\mathcal{B}$. Under the assumption that $(\mathcal{A}, \mathcal{B})$ is stabilizable, $(\mathcal{A}, \mathcal{D})$ is detectable and $g \in L^{2}(0, \tau ; \mathcal{H})$, it is verified in [11] that the Riccati equation

$$
\mathcal{A}^{*} \Pi+\Pi \mathcal{A}+\Pi \mathcal{B} \mathcal{R}^{-1} \mathcal{B}^{*} \Pi+\mathcal{Q}=0
$$

has a unique solution. Furthermore, if $r$ denotes the $\tau$-periodic solution of the adjoint or tracking equation

$$
\begin{aligned}
\dot{r}(t) & =-\left[\mathcal{A}-\mathcal{B} \mathcal{R}^{-1} \mathcal{B}^{*} \Pi\right]^{*} r(t)+\Pi g(t) \\
r(0) & =r(\tau)
\end{aligned}
$$


and $\bar{z}$ is the closed loop solution of

$$
\begin{aligned}
& \dot{\bar{z}}(t)=\left[\mathcal{A}-\mathcal{B} \mathcal{R}^{-1} \mathcal{B}^{*} \Pi\right] \bar{z}(t)-\mathcal{B} \mathcal{R}^{-1} \mathcal{B}^{*} r(t)+g(t) \\
& \bar{z}(0)=\bar{z}(\tau),
\end{aligned}
$$

then the optimal control is given by

$$
\bar{u}(t)=-\mathcal{R}^{-1} \mathcal{B}^{\star}[\Pi \bar{z}(t)-r(t)] .
$$

For implementation purposes, the suboptimal control is computed through approximation techniques analogous to those described in the previous section for the system with no exogenous input. The theoretical extension of the periodic theory for unbounded input operators $\mathcal{B}$ is currently under investigation. The practical efficacy of the method in this regime is demonstrated through numerical examples in the next section.

\section{$5 \quad$ Numerical Examples}

To illustrate the previously described control laws, we consider in this section several numerical examples which demonstrate the attenuation of shell vibrations using surface-mounted piezoceramic actuators. The first example employs the feedback law described in Section 4.1 and demonstrates the capability of the PDE-based controller for attenuating transient vibrations. The second and third examples demonstrate the capability of the period feedback law developed in Section 4.2. The shell is driven by a single frequency source in Example 3 while the attenuation in the case of a multiple frequency input is demonstrated in Example 4.

For all three examples, the exogenous disturbance was constructed to model a periodic noise source localized near the shell center at $\theta=0$ and $\theta=\pi$. To accomplish this, the surface forces were taken to be

$$
\begin{aligned}
& \hat{q}_{x}(t, x, \theta)=\frac{1}{100} q_{x}(t) e^{-20(x-\bar{x})^{2}} \Theta(\theta) \\
& \hat{q}_{\theta}=0 \\
& \hat{q}_{n}(t, x, \theta)=q_{n}(t) e^{-20(x-\bar{x})^{2}} \Theta(\theta)
\end{aligned}
$$

where

$$
\Theta(\theta)= \begin{cases}1-e^{-(\theta-\pi / 2)^{6} / 2} & , \quad 0 \leq \theta<\pi \\ 1-e^{-(\theta-3 \pi / 2)^{6} / 2} & , \quad \pi \leq \theta<2 \pi .\end{cases}
$$

Here $\bar{x}=\ell / 2$ denotes the axial center and $q_{x}(t)$ and $q_{n}(t)$ designate the periodic temporal components to be specified in the examples. The spatial components of the force distributions are illustrated in Figure 4. The normal force in the $x$ and $\theta$ components can be interpreted as modeling periodic pressure sources adjacent to the points $(x, \theta)=(\bar{x}, 0)$ and $(x, \theta)=(\bar{x}, \pi)$.

Twelve pairs of piezoceramic patches were employed as actuators (hence $s=12$ in (1) and (7)) with two sizes considered; the large patches had dimensions of $x=0.2, \theta=\pi / 3$ while the smaller patches had sizes of $x=0.1, \theta=\pi / 6$. As indicated in Figure 5, the patch pairs were configured in sets of three along the lines $\theta=0, \pi / 2, \pi, 3 \pi / 2$. We reiterate that the voltages 
to the inner and outer patches in each pair were independent so as to provide capabilities for generating both inplane forces and bending moments. The dimensions and material properties of the shell and patches are summarized in Table 1.

To resolve the uncontrolled and controlled shell dynamics, the Fourier index $m$ was taken from -4 to 4 for a total of 9 Fourier coefficients. The axial components of the longitudinal and circumferential displacements were approximated using 13 modified cubic splines while 11 splines were employed in the transverse displacement (two degrees of freedom are lost to accommodate the additional zero-slope boundary conditions indicated in $(6))$. This yielded $\mathcal{N}_{u}=\mathcal{N}_{v}=117, \mathcal{N}_{w}=99$ in (19) and a total of $N=666$ coefficients in the ODE system (20).

The important issues of patch number, placement and size fall outside the scope of this work and are under current investigation. The number of patches was fixed in these examples to facilitate comparison and demonstrate the capabilities of the control method under uniform conditions.

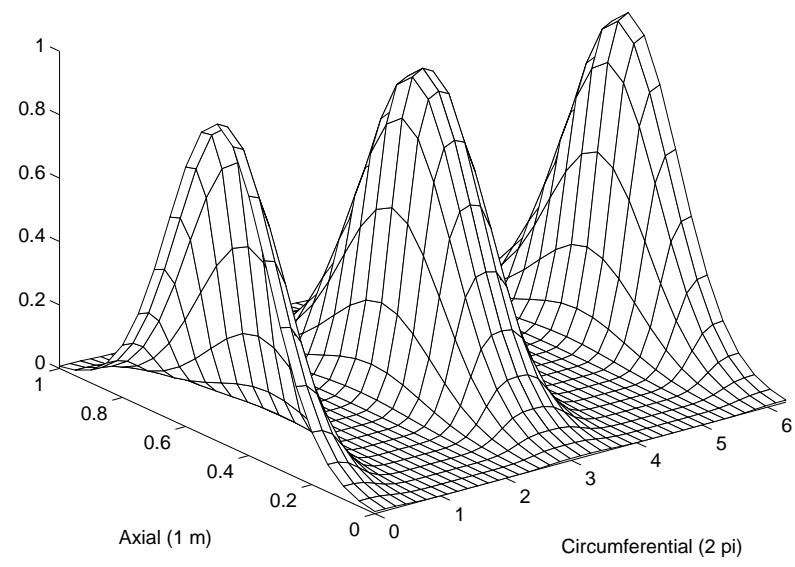

Figure 4. Spatial distribution of transverse and longitudinal forces (26) to the shell.

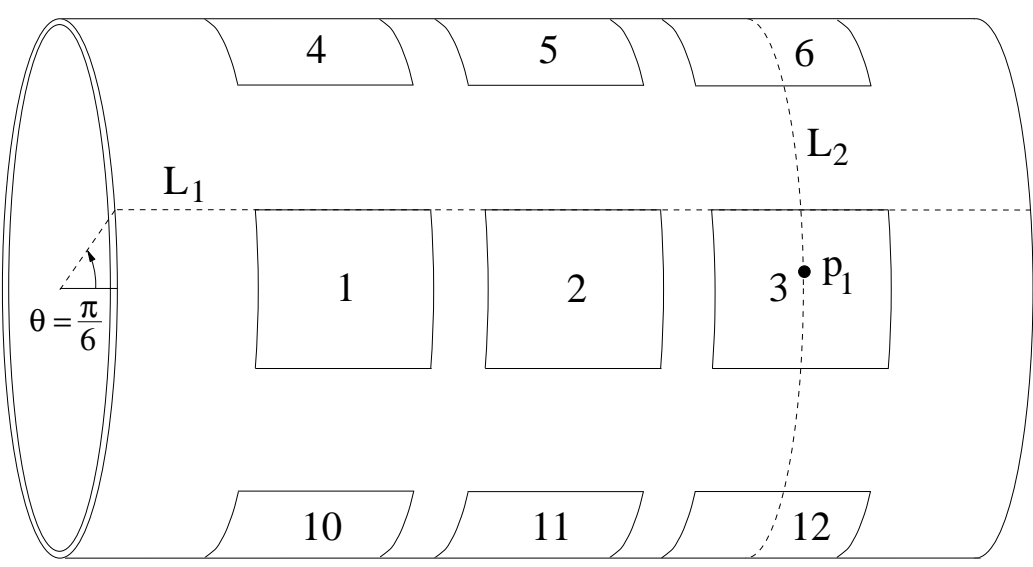

Figure 5. Configuration of large patch pairs 1-6 and 10-12. Pairs 7-9 are centered along $\theta=\pi$. Observation lines $L_{1}=\{(x, \theta) \mid 0 \leq x \leq \ell, \theta=\pi / 6\}, L_{2}=\{(x, \theta) \mid x=3 \ell / 4,0 \leq \theta \leq 2 \pi\}$ and observation point $p_{1}=(\hat{x}, \hat{\theta})=(3 \ell / 4, \pi / 32)$. Configuration of small patches is similar. 


\begin{tabular}{|c|c|c|}
\hline & Dimensions & Parameters \\
\hline Shell & $\begin{array}{l}h=.00127 \mathrm{~m} \\
R=.4 \mathrm{~m} \\
\ell=1 \mathrm{~m}\end{array}$ & $\begin{array}{l}\rho=2700 \mathrm{~kg} / \mathrm{m}^{3} \\
E=7.1 \times 10^{10} \mathrm{~N} / \mathrm{m}^{2} \\
c_{D}=1.47 \times 10^{5} \mathrm{Nms} \\
\nu=.33 \\
\mu=58.97 \mathrm{Ns} / \mathrm{m}^{2}\end{array}$ \\
\hline Patches & $\begin{aligned} h_{p e}=.0001778 m & \\
\text { Centers }(x, \theta): & (.25,0), \quad(.5,0), \quad(.75,0) \\
& (.25, \pi / 2), \quad(.5, \pi / 2), \quad(.75, \pi / 2) \\
& (.25, \pi), \quad(.5, \pi), \quad(.75, \pi) \\
& (.25,3 \pi / 2),(.5,3 \pi / 2),(.75,3 \pi / 2) \\
\text { Dimensions - } & \text { Large Patch: } x: 0.2 \mathrm{~cm}, \theta: \pi / 3 \\
& \text { Small Patch: } x: 0.1 \mathrm{~cm}, \theta: \pi / 6\end{aligned}$ & $\begin{array}{l}\rho_{p e}=7600 \mathrm{~kg} / \mathrm{m}^{3} \\
E_{p e}=6.3 \times 10^{10} \mathrm{~N} / \mathrm{m}^{2} \\
c_{D_{p e}}=1.7 \times 10^{5} \mathrm{Nms} \\
\nu_{p e}=.31 \\
d_{31}=190 \times 10^{-12} \mathrm{~m} / \mathrm{V}\end{array}$ \\
\hline
\end{tabular}

Table 1. Dimensions and physical parameters for the shell and patches.

\section{Example 2.}

We illustrate here the feedback law developed in Section 4.1 with no exogenous force. The shell was initially driven for $t=.01$ seconds by the forces (26) with

$$
q_{x}(t)=q_{n}(t)= \begin{cases}1000 \pi t & , 0 \leq t<.01 \\ 0 & , .01<t \leq .05\end{cases}
$$

at which point the forces were terminated and the shell was allowed to freely vibrate. The open loop response at the point $p_{1}$ as well as the rms displacement values along the lines $L_{1}$ and $L_{2}$ are plotted in Figures $6-8$ (see Figure 5 for the specific locations of $p_{1}, L_{1}$ and $L_{2}$ ). The rms plots illustrate standing waves in all three components of the displacement.

Under approximation, the feedback law from Section 4.1 has the form

$$
u(t)=-R^{-1}\left(B^{N}\right)^{T} \Pi^{N} z^{N}(t)
$$

where $\Pi^{N}$ and $z^{N}(t)$ respectively solve

$$
\left(A^{N}\right)^{T} \Pi^{N}+\Pi^{N} A^{N}+\Pi^{N} B^{N} R^{-1}\left(B^{N}\right)^{T} \Pi^{N}+Q^{N}=0
$$

and

$$
\begin{aligned}
& \dot{z}^{N}(t)=\left[A^{N}-B^{N} R^{-1}\left(B^{N}\right)^{T} \Pi^{N}\right] z^{N}(t) \\
& z^{N}\left(t_{0}\right)=z_{0}
\end{aligned}
$$

(see (20) for the component matrices $A^{N}$ and $B^{N}$ ). For this example, we neglect the passive patch contributions and construct the system matrices using constant parameters $\rho, E, \nu, c_{D}$. As indicated in Example 1, the convergence and exponential closed loop stability of control gains for this case are provided by Theorem 4 . The passive patch contributions are then included in the final two examples. 


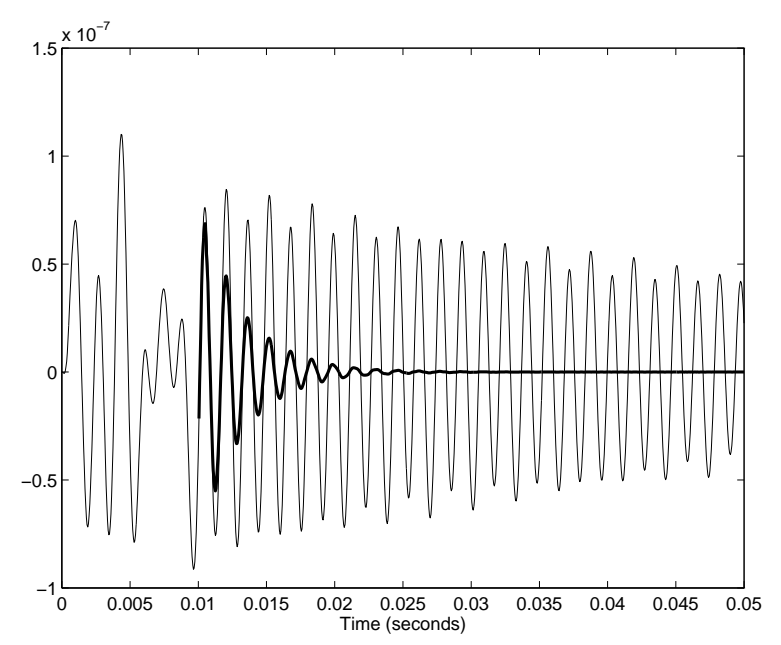

(a)

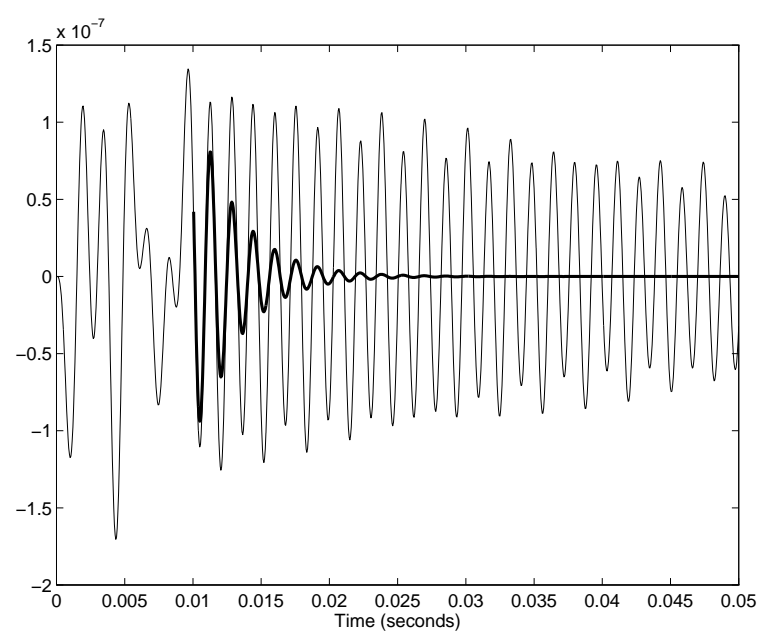

(b)

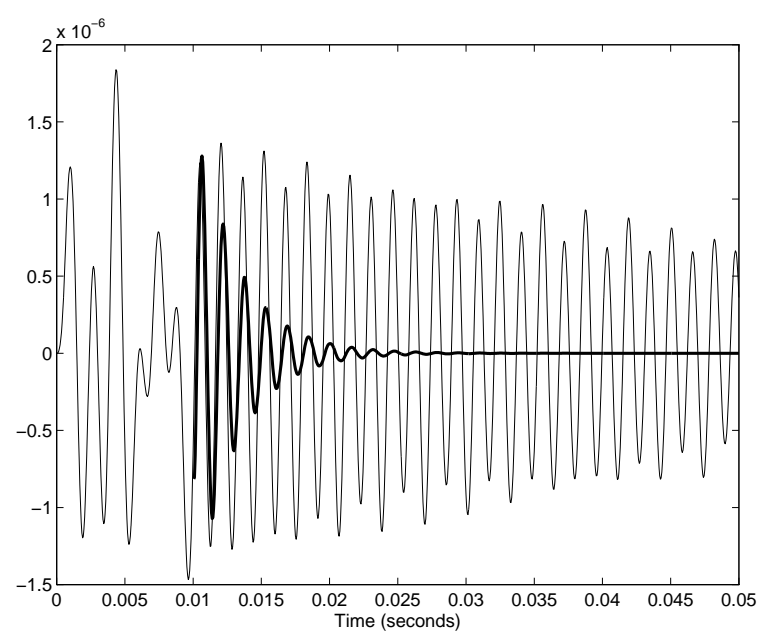

(c)

Figure 6. Uncontrolled and controlled shell displacements at the point $p_{1}$ with control initiated at $t=.01$ using small patches as specified in Table 1 ; (a) longitudinal $u^{N}(t, \hat{x}, \hat{\theta})$, (b) circumferential $v^{N}(t, \hat{x}, \hat{\theta})$, (c) transverse $w^{N}(t, \hat{x}, \hat{\theta}) ;-$ (uncontrolled), - (controlled).

The state and control weights were taken to be

$$
\begin{aligned}
& Q^{N}=\left[\begin{array}{ll}
\mathcal{D}\left(d_{i}\right) & \\
& \mathcal{D}\left(\hat{d}_{i}\right)
\end{array}\right]\left[\begin{array}{ll}
K_{E}^{\mathcal{N}} & \\
& M^{\mathcal{N}}
\end{array}\right], \quad i=1,2,3 \\
& R=\mathcal{D}\left(r_{i}\right) \quad, \quad i=1, \cdots, 24
\end{aligned}
$$

where $M^{\mathcal{N}}$ and $K_{E}^{\mathcal{N}}$ denote the mass and stiffness matrices and $\mathcal{D}\left(d_{i}\right), \mathcal{D}\left(\hat{d}_{i}\right), \mathcal{D}\left(r_{i}\right)$ are diagonal matrices whose $i^{t h}$ component is $d_{i}, \hat{d}_{i}, r_{i}$, respectively. The simulations reported here were computed with the values $d_{i}=\hat{d}_{i}=10^{13}$ and $r_{i}=50$. Large values of $d_{i}, \hat{d}_{i}$ are not uncommon for such systems (e.g., see [2]) and simulations have been run with values as large as $d_{i}=\hat{d}_{i}=10^{20}$ without experiencing degradation due to conditioning. Finally, the choice of a weighted mass matrix for the design matrix $Q^{N}$ is motivated by energy considerations as detailed in [3]. 
The closed loop dynamics of the shell were computed by numerically integrating the ODE system (28) over the time interval $[.01, .05]$. The time history of the component displacements at the point $p_{1}$ for the smaller $(.1, \pi / 6)$ patches are plotted in Figure 6 . Corresponding time trajectories with larger $(.2, \pi / 3)$ patches are plotted in Figure 7 with corresponding rms values along the lines $L_{1}$ and $L_{2}$ plotted in Figure 8 . The time histories illustrate that while substantial control is obtained with the smaller patches, the attenuation is enhanced significantly through the use of larger patches. In this latter case, the vibrations in all components are completely attenuated by time $T=.015$ seconds. The rms plots demonstrate that strong attenuation is attained across the shell including regions not covered by patches. This illustrates both the capabilities of the patches as well as the issues associated with patch size. The larger patches will be employed throughout the remaining examples. Questions regarding patch size, placement and number are under current investigation.

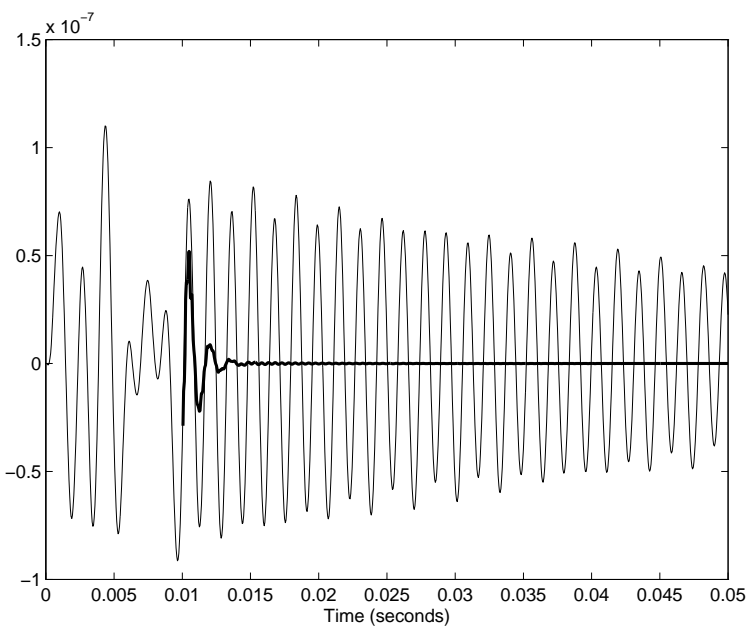

(a)

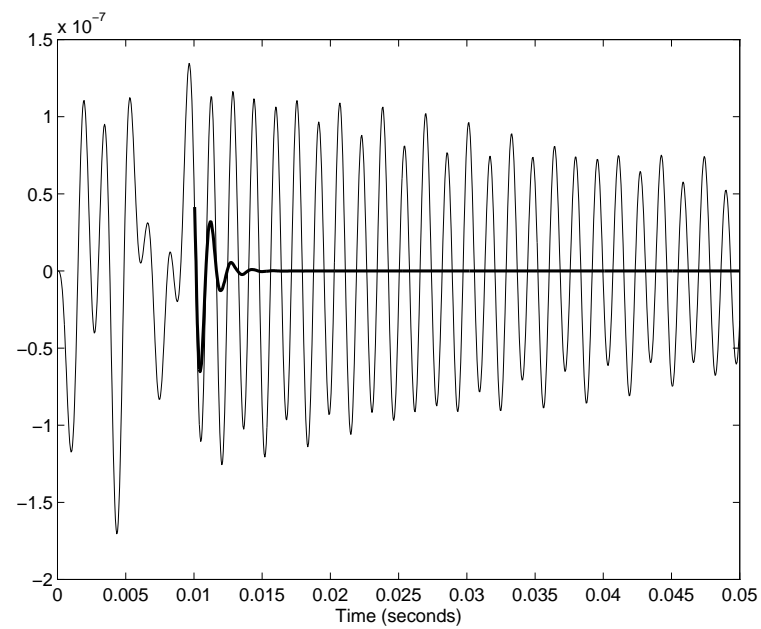

(b)

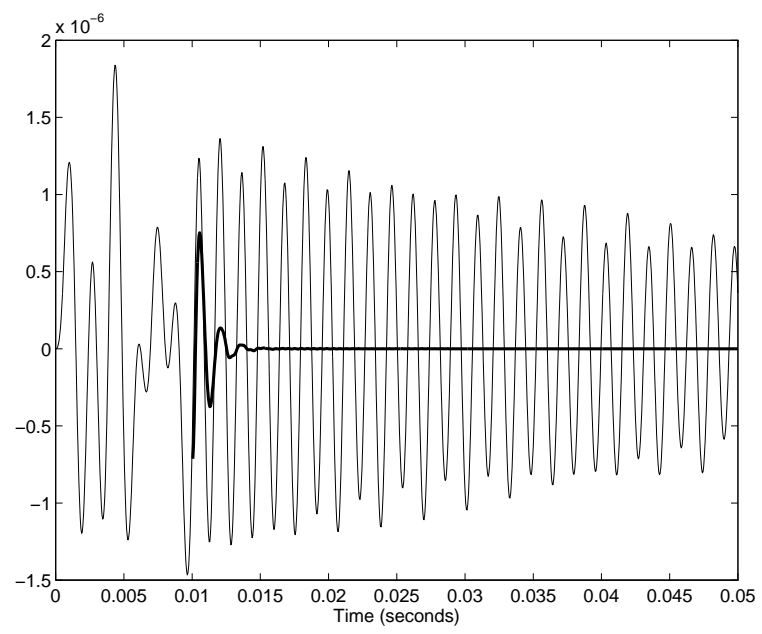

(c)

Figure 7. Uncontrolled and controlled shell displacements at the point $p_{1}$ with control initiated at $t=.01$ using large patches as specified in Table 1; (a) longitudinal $u^{N}(t, \hat{x}, \hat{\theta})$, (b) circumferential $v^{N}(t, \hat{x}, \hat{\theta}),\left(\right.$ c) transverse $w^{N}(t, \hat{x}, \hat{\theta}) ;-$ (uncontrolled), - (controlled). 

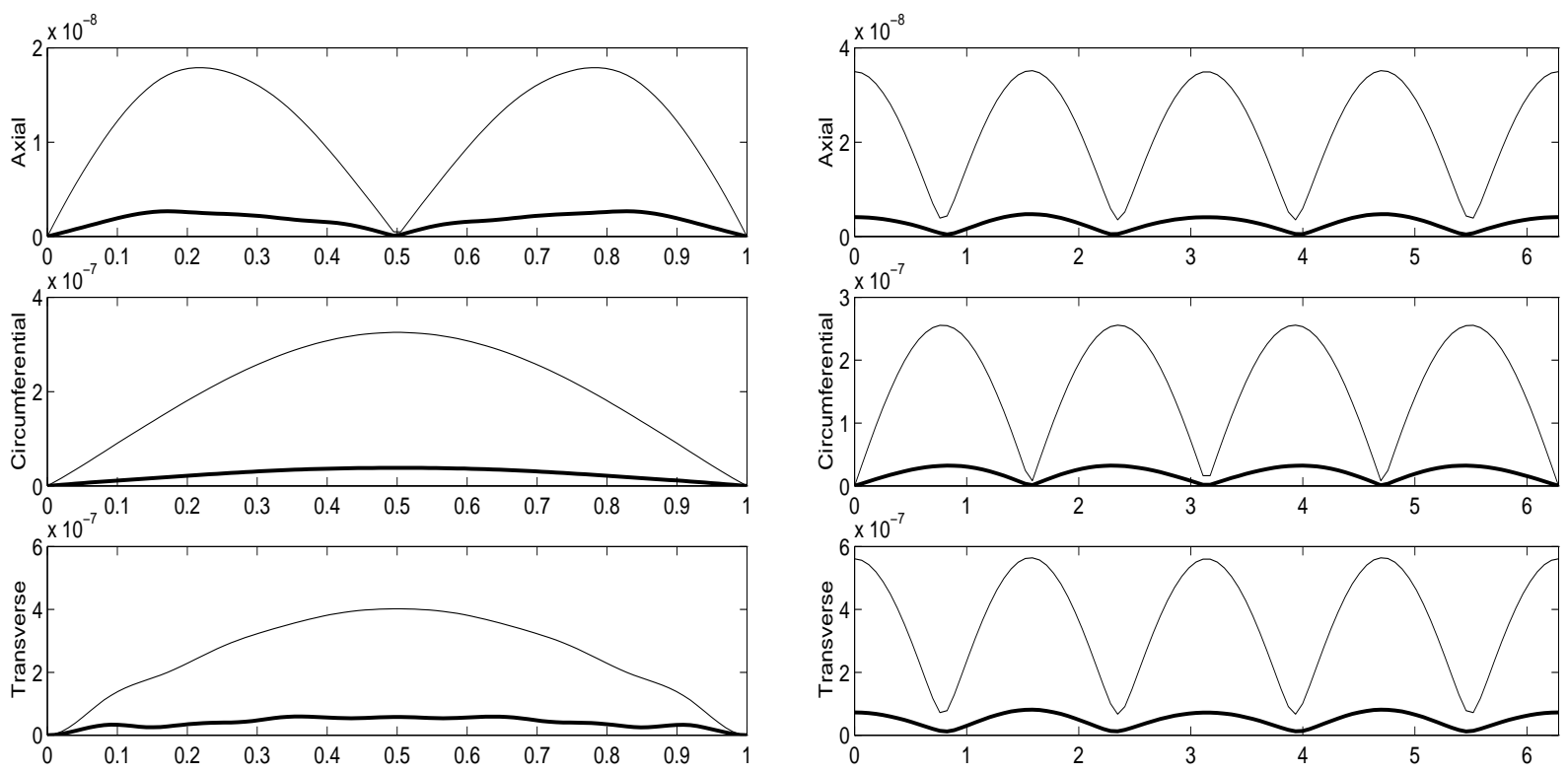

(a)

(b)

Figure 8. Root mean square (rms) displacements with control using large patches; (a) axial line $L_{1}$ and (b) circumferential line $L_{2} ;-$ (uncontrolled), - (controlled).

\section{Example 3.}

The remaining examples illustrate the feedback law of Section 4.2 which accommodates a periodic exogenous disturbance $g$. For this example, a single frequency $500 \mathrm{~Hz}$ temporal input

$$
q_{x}(t)=q_{n}(t)=100 \sin (1000 \pi t)
$$

was employed in the exogenous forces (26).

The approximate control law for this case was computed in the following manner. The adjoint solution $r^{N}(t)$ was computed by numerically integrating the system

$$
\begin{aligned}
& \dot{r}^{N}(t)=-\left[A^{N}-B^{N} R^{-1}\left(B^{N}\right)^{T} \Pi^{N}\right]^{T} r^{N}(t)+\Pi^{N} g^{N}(t) \\
& r^{N}(\tau)=0
\end{aligned}
$$

from the future time $\tau$ back to the present (the Riccati solution satisfies (27)). The optimal control and closed loop solution were then obtained via

$$
u(t)=-R^{-1}\left(B^{N}\right)^{T}\left[\Pi^{N} z^{N}(t)-r^{N}(t)\right]
$$

and

$$
\begin{aligned}
& \dot{z}^{N}(t)=\left[A^{N}-B^{N} R^{-1}\left(B^{N}\right)^{T} \Pi^{N}\right] z^{N}(t)-B^{N} R^{-1}\left(B^{N}\right)^{T} r^{N}(t)+g^{N}(t) \\
& z^{N}(0)=0 .
\end{aligned}
$$

To illustrate the effects of passive (material) patch contributions, we consider two cases. In the first, passive contributions are neglected, as in Example 2, and system matrices are constructed using constant parameters $\rho, E, \nu, c_{D}$. In the second case, all passive contributions are retained in the resultants (2), (3) and density expressions, and hence are included in the system matrices $A^{N}$ and vectors $B^{N}, g^{N}$ through the inverted mass matrix. 
The uncontrolled and controlled dynamics at the point $p_{1}$ for the first case are plotted in Figure 9 while corresponding plots for the system which includes passive properties are given in Figure 10. In both cases, the time intervals are restricted to $[0, .05]$ to illustrate the system dynamics. A comparison indicates that the inclusion of passive or material properties has a significant effect on both open and closed loop dynamics due to the additional density, stiffness and damping provided by the patches. Hence for many systems, it is necessary to incorporate these components to attain an accurate model and a feasible model-based control method (see $[10,14]$ for experimental evidence demonstrating the necessity of including passive patch components). From a control perspective, it is noted that for this patch configuration, transverse vibrations are reduced significantly throughout the shell with attenuation levels of approximately $80 \%$ at the point $p_{1}$. Similar attenuation is noted in the longitudinal and circumferential components. The spatial nature of the uncontrolled and controlled shell in this regime is indicated by the rms values along the axial line $L_{1}$ and circumferential line $L_{2}$ as plotted in Figure 11. These plots illustrate that in spite of the persistent exogenous force, significant attenuation is attained in all components of the displacement.

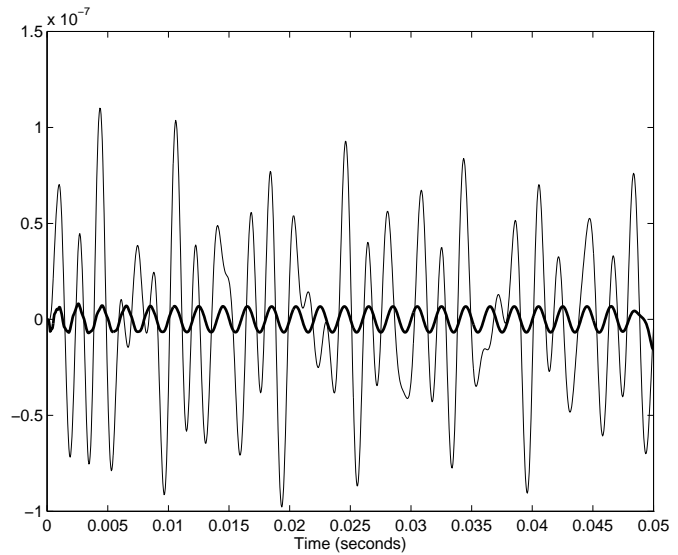

(a)

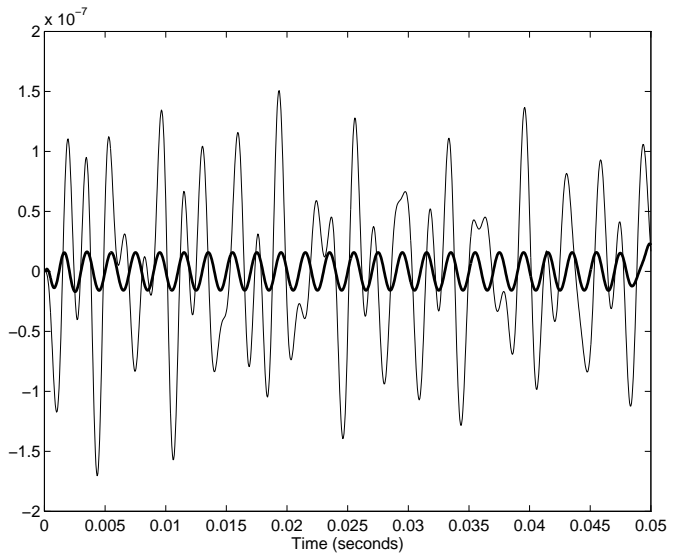

(b)

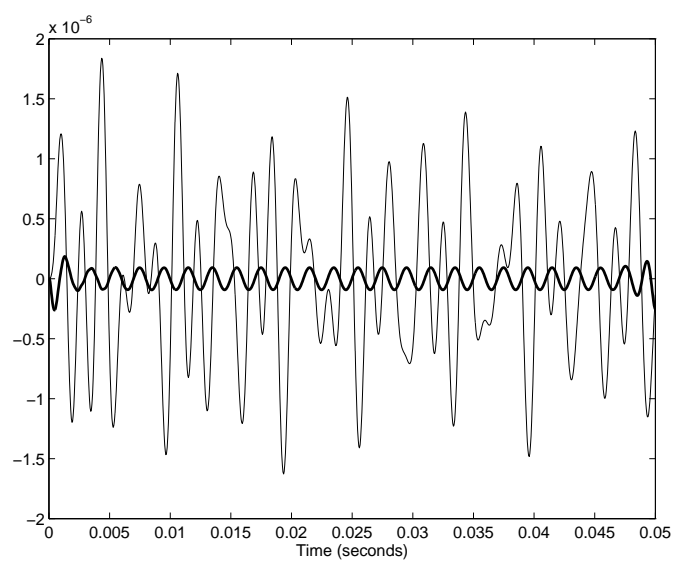

(c)

Figure 9. Uncontrolled and controlled displacements at the point $p_{1}$ without passive patch components included in system matrices; (a) longitudinal $u^{N}(t, \hat{x}, \hat{\theta})$, (b) circumferential $v^{N}(t, \hat{x}, \hat{\theta}),(\mathrm{c})$ transverse $w^{N}(t, \hat{x}, \hat{\theta}) ;-$ (uncontrolled), (controlled). 


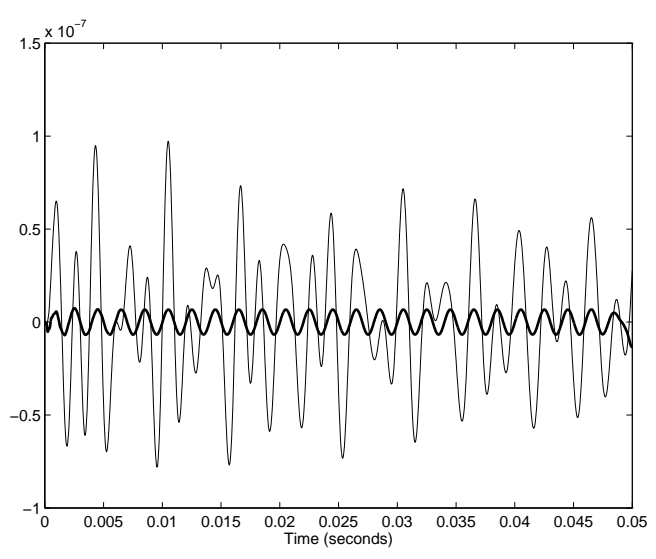

(a)

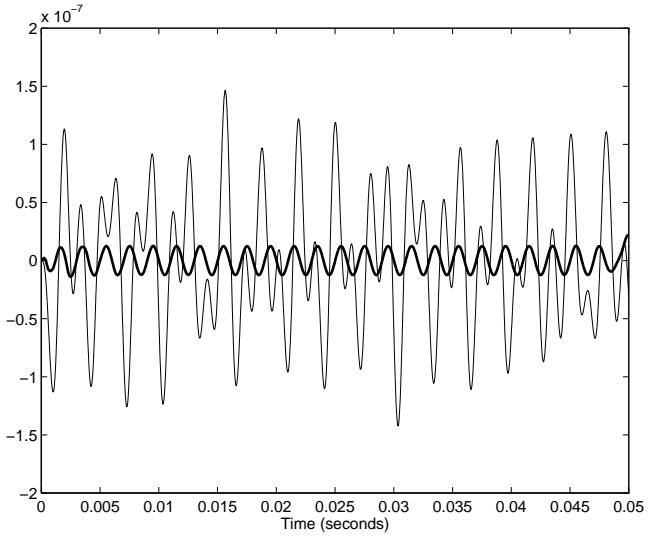

(b)

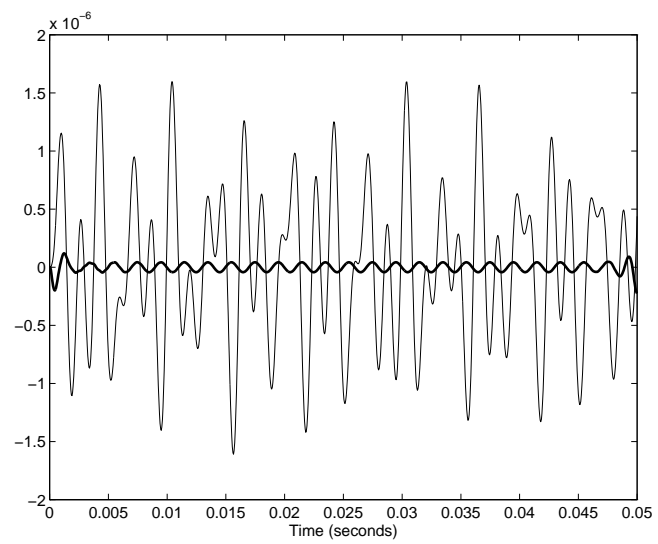

(c)

Figure 10. Uncontrolled and controlled displacements at the point $p_{1}$ with passive patch components included in system matrices; (a) longitudinal $u^{N}(t, \hat{x}, \hat{\theta})$, (b) circumferential $v^{N}(t, \hat{x}, \hat{\theta}),(\mathrm{c})$ transverse $w^{N}(t, \hat{x}, \hat{\theta}) ;-$ (uncontrolled), (controlled).
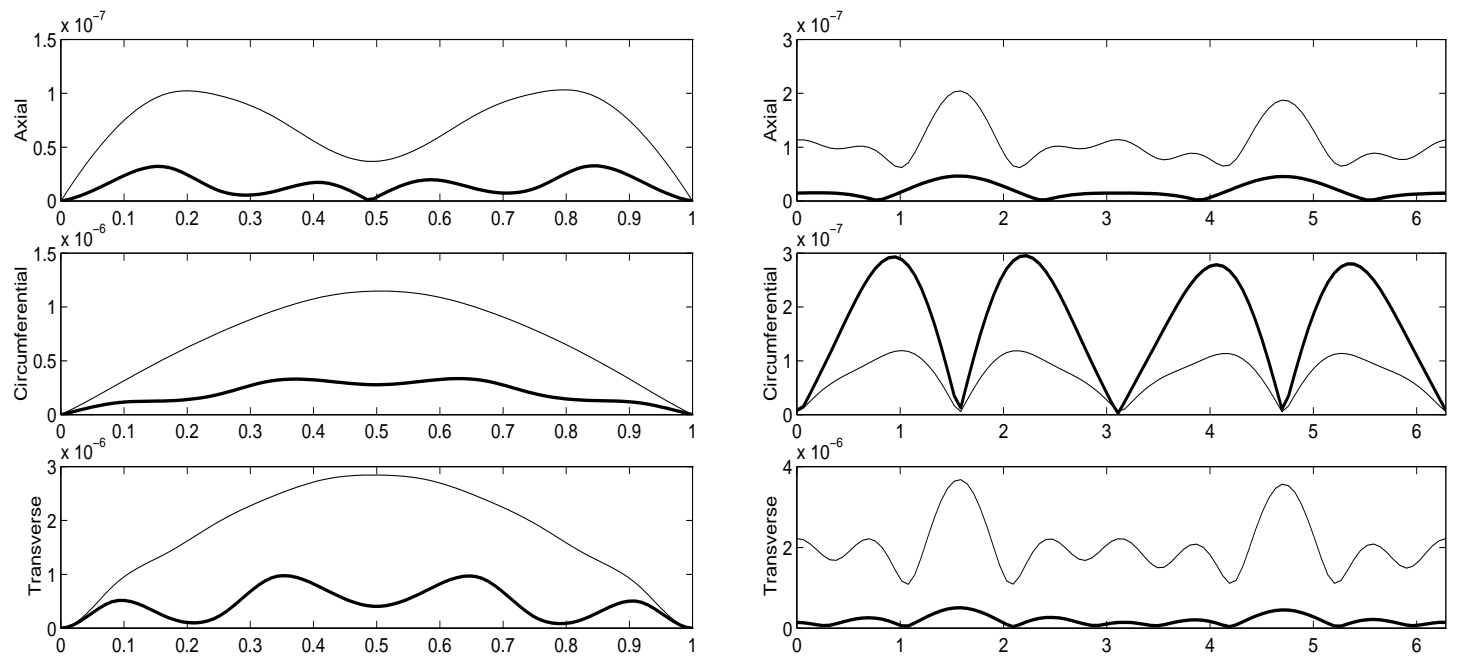

(a)

(b)

Figure 11. Root mean square (rms) displacements with passive patch components included in system; (a) axial line $L_{1}$ and (b) circumferential line $L_{2} ;-$ (uncontrolled), - (controlled). 


\section{Example 4.}

This final example demonstrates that the performance of the controller is not degraded by a larger number of frequencies in the exogenous disturbance and hence structural response. In this case, we employed

$$
q_{x}(t)=q_{n}(t)=50[\sin (320 \pi t)+\sin (440 \pi t)+\sin (600 \pi t)+\sin (660 \pi t)+\sin (820 \pi t)]
$$

so that the exogenous disturbance has five frequencies ranging from $160 \mathrm{~Hz}$ to $410 \mathrm{~Hz}$.

The closed loop solution computed using the feedback law (29) is compared with the uncontrolled solution in Figure 12 and 13. The temporal trajectories at the point $p_{1}$ reflect the multiple frequencies in the exogenous disturbance and illustrate that the feedback law (29) is highly effective for such broadband responses. This observation is reinforced by the rms plots in Figure 13 which indicate significant reductions along the lines $L_{1}$ and $L_{2}$.

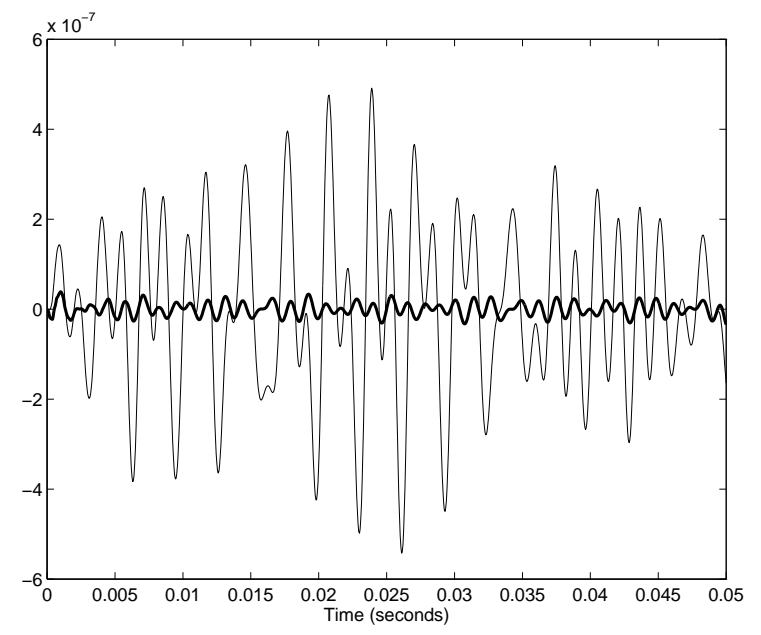

(a)

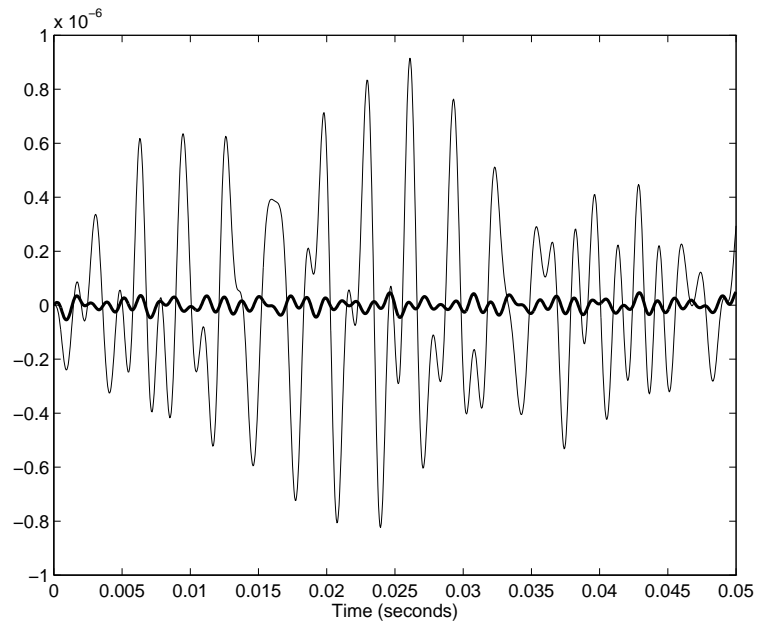

(b)

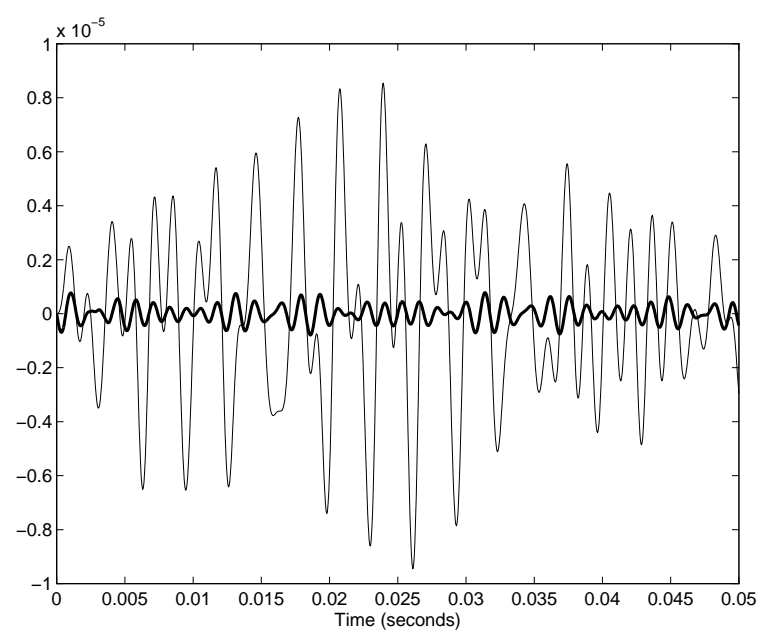

(c)

Figure 12. Uncontrolled and controlled shell displacements at the point $p_{1}$; (a) longitudinal $u^{N}(t, \hat{x}, \hat{\theta})$, (b) circumferential $v^{N}(t, \hat{x}, \hat{\theta})$, (c) transverse $w^{N}(t, \hat{x}, \hat{\theta}) ;-$ (uncontrolled), (controlled). 
The voltages given by (29) for patch pairs 1-6 are plotted in Figure 14 (the outputs from the remaining patch pairs are identical due to symmetry). Due to the axial symmetry of the transverse force $\hat{q}_{n}$, the voltages to patch pairs 1 and 3,4 and 6 are nearly identical. It is also noted that the voltages to the central patch pairs 2 and 5 are larger than those to surrounding pairs to accommodate the larger axial force distribution delivered to the shell center. The predominance of the circumferential force near $\theta=0, \pi$ leads to larger voltages to patch pairs 1-3 than 4-6. A comparison of the voltages to the inner and outer patches in each pair first illustrates that the voltages are nearly diametrically out-of-phase which leads to the generation of significant bending moments (see (5)). As indicated in Figure 15, the voltages also exhibit magnitude differences and hence also generate inplane forces in addition to the bending moments. The small magnitude of the inplane voltages, as compared with the total voltages plotted in Figure 14, illustrates the degree to which bending motion dominates inplane motion for this force configuration. Finally, it should be noted that the voltages to the patches are less than $80 \mathrm{~V}$ rms which is physically reasonable for patches having the specified thickness. The combination of these effects provides the controller with significant vibration attenuation capabilities for shell systems excited by general inputs.
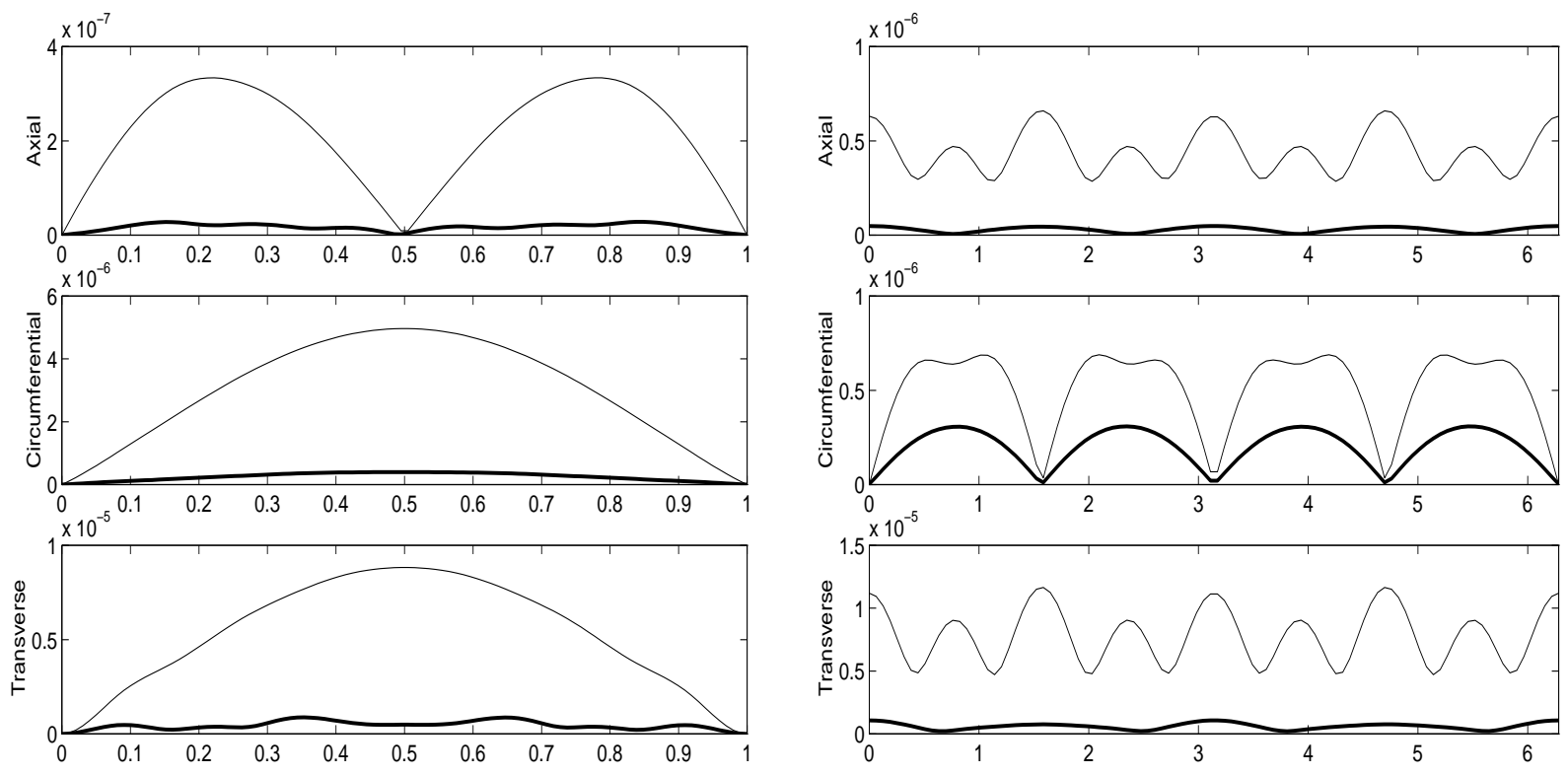

(a)

(b)

Figure 13. Root mean square (rms) displacements; (a) axial line $L_{1}$ and (b) circumferential line $L_{2} ;-$ (uncontrolled), (controlled). 


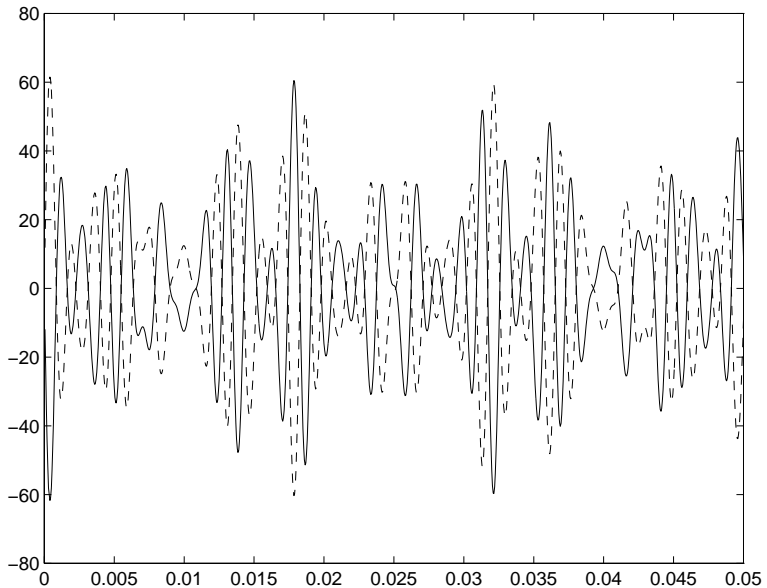

(a) Pair 1

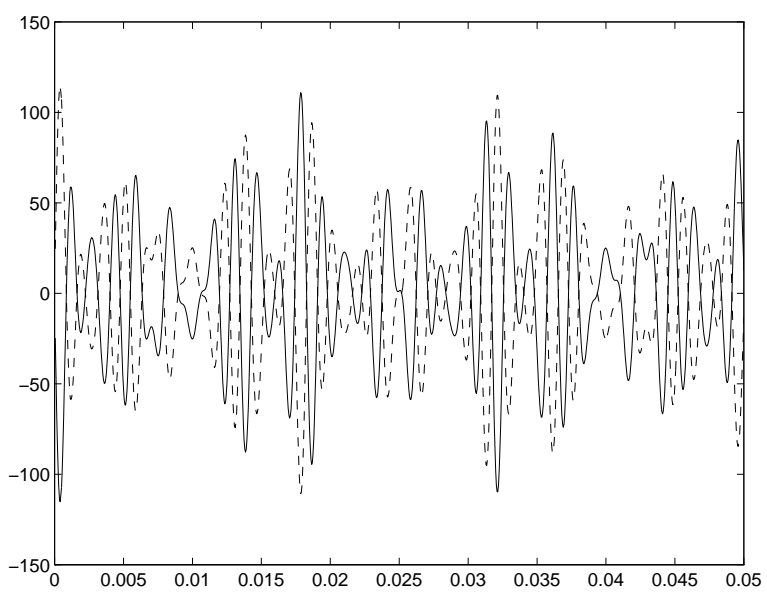

(b) Pair 2

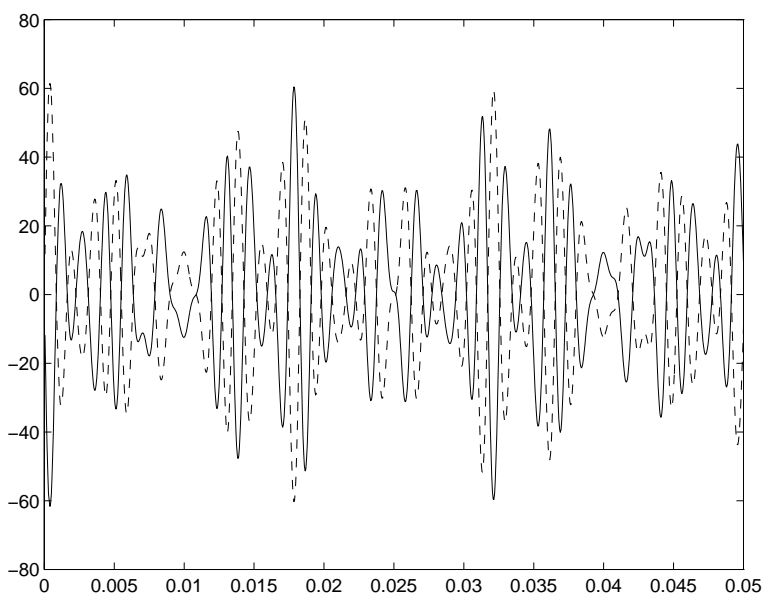

(c) Pair 3

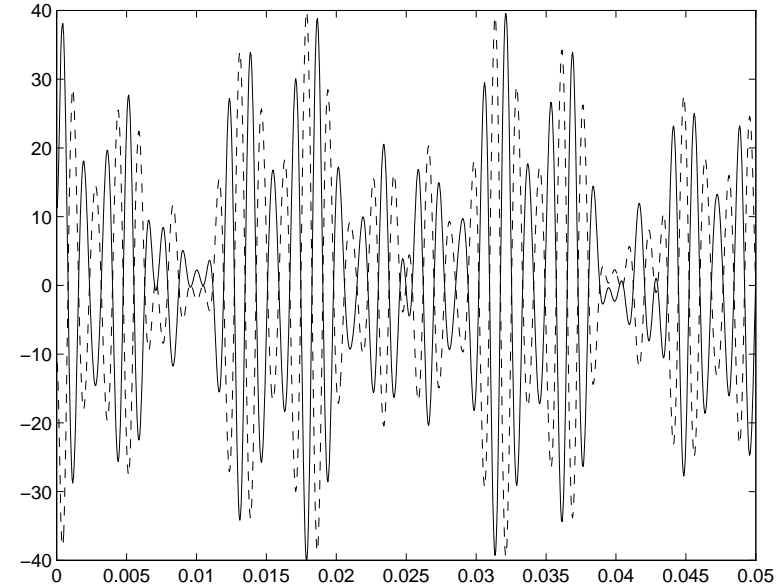

(d) Pair 4

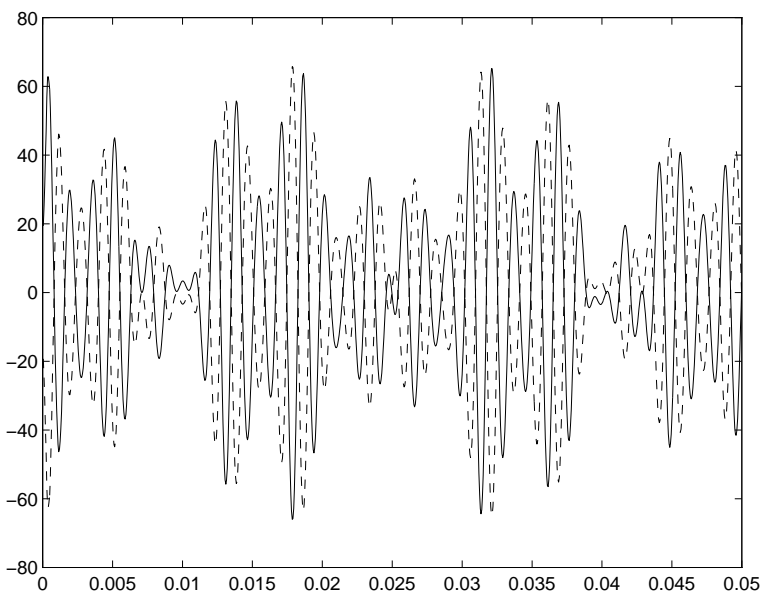

(e) Pair 5

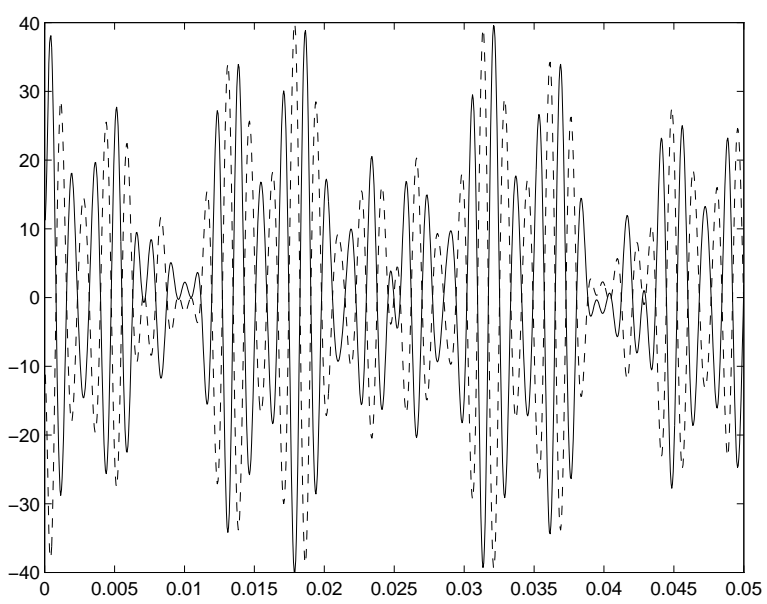

(f) Pair 6

Figure 14. Voltages to the inner and outer patches of pairs 1-6 as depicted in Figure 5. (a) Pair 1, (b) Pair 2, (c) Pair 3, (d) Pair 4, (e) Pair 5, (f) Pair 6; ——outer patch, - - inner patch. 


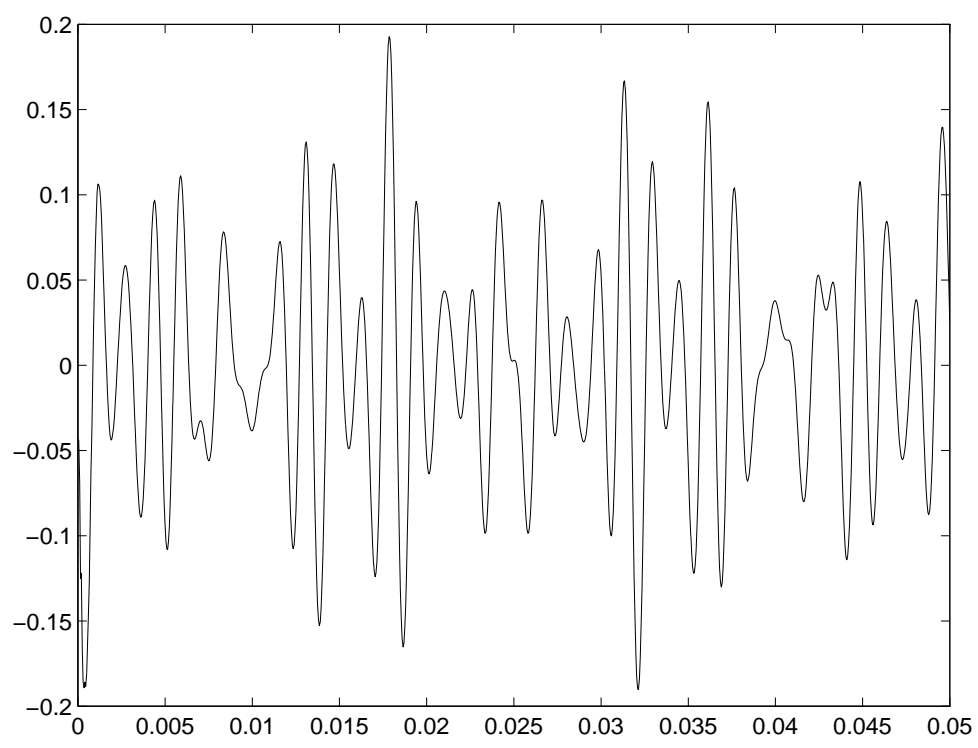

Figure 15. Sum of inplane voltages to patch pair 1.

\section{Conclusions}

This work summarizes issues concerning the formulation and numerical implementation of a PDE-based LQR control method for thin cylindrical shells with surface-mounted piezoceramic actuators. From a physical perspective, such actuators are popular since they are lightweight, relatively inexpensive and provide broadband control inputs. When mounted in pairs on a cylindrical shell, they can be used to generate both inplane forces and bending moments which provides the capability for controlling the coupled longitudinal, circumferential and transverse vibrations of the structure.

Controller design for thin cylindrical shells is significantly more difficult than for plates or beams due to the complexity of the structures and the inherent coupling of component displacements due to curvature. The models, numerical methods and control laws must incorporate this coupling to be fully effective in applications involving shell-like structures.

In this work, Donnell-Mushtari equations modified to incorporate passive and active patch contributions were used to characterize the shell dynamics. This model incorporates the primary coupling mechanisms while remaining sufficiently simple to permit initial analysis of the control methods. As detailed in [10, 15, 21, 26], the model can be directly extended to attain higher accuracy if the application warrants.

A Galerkin method utilizing bases constructed from tensored Fourier polynomials and modified cubic splines was used to approximate the system dynamics. As discussed in $[1,20]$, an important issue when approximating shell dynamics concerns the development of methods which avoid shear or membrane locking. One manifestation of locking is the existence of model dynamics which are incorrectly approximated by the numerical method. The use of a numerical method which exhibits locking can lead to a loss of control authority and potential controller destabilization if the approximations are sufficiently inaccurate. As detailed in [12], 
the Galerkin method employed here avoids locking and provides highly accurate approximate solutions with moderate discretization levels.

The LQR control method discussed here accommodates the unbounded input operators due to the discrete nature of the patches. A well-posedness and convergence framework is obtained through the abstract formulation of the control problem in terms of sesquilinear forms and associated linear operators. Because the LQR method requires full state knowledge for computation of control voltages to the patches, it cannot be directly implemented in experiments. It does, however, provide a means for quantifying optimal patch capabilities and performance and a setting in which to investigate questions concerning patch placement, number, et cetera. It also provides the system and input matrices necessary for later construction of a state estimator and compensator which can be experimentally implemented [10].

The numerical examples demonstrate the performance of the method for a variety of exogenous disturbances. Specifically, they illustrate that through the incorporation of coupling mechanisms in the PDE model and hence controller, optimal patch voltages are determined which significantly attenuate all three components of the shell displacement. Furthermore, by modeling the global shell dynamics and patch interactions through coupled PDE and constructing the control laws in terms of these PDE, significant reductions in both transient and periodic displacements are obtained throughout the shell, including regions which are devoid of patches. This illustrates the optimal attenuation possible for the given patch configuration and operating conditions.

An important issue which lies beyond the scope of this paper concerns the optimal number, size and placement of patches for a given set of operating conditions. These issues transcend the specified control method described here and must be addressed when designing any piezoceramic-based control method for the system. The advantage of the PDE-based method for addressing these problems lies in the incorporation of basic physical principles in the models and the utilization of these principles in the control laws.

\section{Acknowledgements}

The authors thank H.T. Banks, CRSC, North Carolina State University, for input regarding various aspects of this investigation.

\section{References}

[1] I. Babuška and M. Suri, "Locking Effects in the Finite Element Approximation of Elasticity Problems," Numerische Mathematik, 62, pp. 439-463, 1992.

[2] H.T. Banks, M.A. Demetriou and R.C. Smith, "An $H^{\infty} /$ MinMax Periodic Control in a 2-D Structural Acoustic Model with Piezoceramic Actuators," IEEE Transactions on Automatic Control, 41(7), pp. 943-959, 1996.

[3] H.T. Banks, W. Fang, R.J. Silcox and R.C. Smith, "Approximation Methods for Control of Acoustic/Structure Models with Piezoceramic Actuators," Journal of Intelligent Material Systems and Structures, 4(1), pp. 98-116, 1993. 
[4] H.T. Banks and K. Ito, "A Unified Framework for Approximation in Inverse Problems for Distributed Parameter Systems," Control-Theory and Advanced Technology, 4, pp. 73-90, 1988 .

[5] H.T. Banks and K. Ito, "Approximation in LQR Problems for Infinite Dimensional Systems with Unbounded Input Operators," CRSC Technical Report CRSC-TR94-22, November 1994; Journal of Mathematical Systems, Estimation and Control, 7, pp. 119122 (Abstract), 1997.

[6] H.T. Banks, Z-H. Luo, L.A. Bergman and D.J. Inman, "On the Existence of Normal Modes of Damped Discrete-Continuous Systems," CRSC Technical Report CRSC-TR975, February 1997; ASME Journal of Applied Mechanics, submitted.

[7] H.T. Banks, R.C. Smith, D.E. Brown, V.L. Metcalf and R.J. Silcox, "The Estimation of Material and Patch Parameters in a PDE-Based Circular Plate Model," Journal of Sound and Vibration, 199(5), pp. 777-799, 1997.

[8] H.T. Banks, R.C. Smith and Y. Wang, "The Modeling of Piezoceramic Patch Interactions with Shells, Plates, and Beams," Quarterly of Applied Mathematics, 53(2), pp. 353-381, 1995.

[9] H.T. Banks, R.C. Smith and Y. Wang, "Modeling and Parameter Estimation for an Imperfectly Clamped Plate," Computation and Control IV, (K.L. Bowers and J. Lund, eds.), Birkhäuser, Boston, pp. 23-42, 1995.

[10] H.T. Banks, R.C. Smith and Y. Wang, Smart Material Structures: Modeling, Estimation and Control, Masson/John Wiley, Paris/Chichester, 1996.

[11] G. Da Prato, "Synthesis of Optimal Control for an Infinite Dimensional Periodic Problem," SIAM Journal of Control and Optimization, 25(3), pp. 706-714, 1987.

[12] R. del Rosario and R.C. Smith, "Spline Approximation of Thin Shell Dynamics," International Journal for Numerical Methods in Engineering, 40, pp. 2807-2840, 1997.

[13] C.R. Fuller, S.D. Snyder, C.H. Hansen and R.J. Silcox, "Active Control of Interior Noise in Model Aircraft Fuselages Using Piezoceramic Actuators," Paper 90-3922, AIAA 13th Aeroacoustics Conference, Tallahassee, FL, October 1990.

[14] S. Griffin and B. Henderson, "An Investigation into Inaccuracies in Closed-Form Piezoceramic Actuator Models for Smart Structures," Proceedings of the 38th AIAA/ ASME/ASCE/AHS/ASC Structures, Structural Dynamics, and Materials Conference and Exhibit, AIAA/ASME/AHS Adaptive Structures Forum, AIAA 97-1309, Kissimmee, FL, April 7-10, 1997.

[15] H. Kraus, Thin Elastic Shells: An Introduction to the Theoretical Foundations and the Analysis of Their Static and Dynamic Behavior, John Wiley and Sons, Inc., New York, 1967. 
[16] I. Lasiecka, "Finite Element Approximations of Compensator Design for Analytic Generators with Fully Unbounded Controls/Observations," SIAM Journal of Control and Optimization, 33(1), pp. 67-88, 1995.

[17] I. Lasiecka and R. Marchand, "Control and Stabilization in Nonlinear Structural Acoustic Problems," Proceedings of Fourth Annual SPIE Symposium on Smart Structures and Materials, San Diego, CA, March 2-6, 1997, to appear.

[18] I. Lasiecka and R. Marchand, "Riccati Equations Arising in Acoustic Structure Interactions with Curved Walls," Journal of Dynamics and Control, submitted.

[19] I. Lasiecka and R. Triggiani, "Numerical Approximations of Algebraic Riccati Equations for Abstract Systems Modelled by Analytic Semigroups, and Applications," Mathematics of Computation, 57(196), pp. 639-662 and S13-S37, 1991.

[20] Y. Leino and J. Pitkäranta, "On the Membrane Locking of $h-p$ Finite Elements in a Cylindrical Shell Problem," International Journal for Numerical Methods in Engineering, 37, pp. 1053-1070, 1994.

[21] A.W. Leissa, Vibration of Shells, NASA SP-288, 1973; Reprinted by the Acoustical Society of America through the American Institute of Physics, 1993.

[22] C. McMillan, "Uniform Stabilization of a Thin Cylindrical Shell," Dynamics of Continuous, Discrete and Impulsive Systems, submitted.

[23] A. Pazy, Semigroups of Linear Operators and Applications to Partial Differential Equations, Springer-Verlag, New York, 1983.

[24] R.E. Showalter, Hilbert Space Methods for Partial Differential Equations, Pitman Publishing Ltd., London, 1977.

[25] R.J. Silcox, S. Lefebvre, V.L. Metcalf, T.B. Beyer and C.R. Fuller, "Evaluation of Piezoceramic Actuators for Control of Aircraft Interior Noise," Proceedings of the DGLR/AIAA 14th Aeroacoustics Conference, Aachen, Germany, May 11-14, 1992.

[26] W. Soedel, Vibrations of Shells and Plates, Second Edition, Marcel Dekker, Inc., New York, 1993.

[27] V.R. Sonti and J.D. Jones, "Dynamic Effects of Piezoactuators on the Cylindrical Shell Response," AIAA Journal, 34(4), pp. 795-801, 1996.

[28] H. Tanabe, Equations of Evolution, Pitman Publishing Ltd., London, 1979. 